\title{
Basic models in epidemiology
}

Fred Brauer

Department of Mathematics

University of Wisconsin

Madison, Wisconsin

USA 53706
Carlos Castillo-Chavez

Biometrics Unit

Cornell University

Ithaca, New York

USA 14853 


\title{
Basic models in epidemiology
}

\author{
Fred Brauer ${ }^{\dagger}$ and Carlos Castillo-Chavez*
}

\begin{abstract}
This chapter gives an introduction to epidemiological models for homogeneously mixing populations. A flexible framework for modeling deterministic and stochastic--continuous time Markov processes-- pair-formation processes is revisited. This framework is used to formulate demographic models for heterogeneous mixing populations which can be used in the construction of models for communicable and sexually-transmitted diseases. Extensions to stochastic epidemiological processes are outlined.
\end{abstract}

1991 Mathematics Subject Classification: 92D30.

\section{Introduction}

There has been a long debate on whether or not chaotic dynamics occur in natural populations (see Hastings et al. 1993). The controversy arises because various natural populations exhibit apparently "random" fluctuations in abundance. Furthermore, in several instances these fluctuations will look random not only through the naked eye but also through the "eyes" of some standard timeseries analysis (see Ellner 1989). Several simple nonlinear mathematical ecological models that exhibit chaos for apparently reasonable parameter values have been studied over the last few years . Initially chaos was found in simple discrete time models for single species (May 1974; May 1976; May and Oster 1976). Chaotic dynamics have been also encountered in discrete models with age-structure (Ebenman 1987; Guckenheimer et al. 1977; Levin 1981; Levin and Goodyear 1980); in discrete models with two species (Allen 1989a, b; Allen 1990a, b; Allen 1991; Beddington et al. 1975; Bellows and Hassell 1988; May 1974; May and Oster 1976; Neubert and Kot 1992); in simple discrete models of parasites (May 1985); in models for host-parasitoid-pathogen systems (Hochberg et al. 1990); in discrete demographic models with two sexes (Caswell and Weeks 1986); and in discrete models that include frequency dependent selection (Altenberg 1991; Cressman 1988; May and Anderson 1983a). Continuous-time

$\dagger$ Department of Mathematics, University of Wisconsin, Madison, WI 53706.

* To whom correspondence should be sent.

* Biometrics Unit and Center for Applied Mathematics, 337 Warren Hall, Cornell University, Ithaca, NY 14853-7801. 
ecological models that exhibit chaos have also been found in Lotka-Volterra type models (Gilpin 1992; Schaffer 1985a, b; Gardini et al. 1987; Takeuchi and Adachi 1983) and in three species food web chain models (Hasting and Powell 1991). Chaos has also been found in epidemic models (Kot et al. 1988; Olsen and Schaffer 1990; Schaffer 1985a; Schaffer and Kot 1985; Schaffer et al. 1990). Reviews that include many more models that have exhibited chaos in ecology include those of May (1987) and Hastings et al. (1993), the organizing source of the above references. In summary, simple and strategic ecological models can exhibit chaotic dynamics for what appears to be likely regions of parameter space, and current research focuses on increasing our understanding of the mechanisms that are more likely to lead to chaos as well as their implications in theoretical biology.

The study of chaos in ecology through the use of strategic models must be complemented with the analysis of time series because some parameters estimates will have errors, other parameters will not be measurable, and our results will be model dependent. Several time series methods have been used to detect chaos in epidemiological data including some based on dynamical systems theory-model-free approaches (Kot et al. 1988; Olsen and Schaffer 1990; Schaffer 1985a; Schaffer and Kot 1985; Ellner 1989; Schaffer et al. 1990). Reviews of model-free and model based approaches are discussed in Hastings et al. (1993) and Ellner (1989). Because of the extensive use of epidemiological data in combination with dynamical systems theory to study the presence of chaos in real epidemiological systems, it has become important to gain some understanding of epidemic modeling. The rest of this chapter concentrates on a brief introduction to epidemic theory.

Epidemiological models have been used to study ecological and epidemiological phenomena extensively (see Anderson 1982; Anderson and May 1991; Bailey 1975; Busenberg and Cooke 1993; Capasso 1993; Castillo-Chavez 1989; Gabriel et al. 1990; Hethcote and Yorke 1984; Hethcote and Van Ark 1992, Jewell et al. 1991; Kaplan and Brandeau 1994; and references therein). Their success is partly based on the fact that they provide an ideal tool to model tight coevolutionary interactions (see Levin 1983b, Levin and Castillo-Chavez, 1989 and references therein), that is, interactions in which the fate of the host (the resource) is intimately connected to the fate of the pathogen. Implicitly, hosts are modeled as dynamic patches (e. g. with their own life history) that are subject to invasions (by pathogens). These invasions affect temporarily or permanently the quality (even the life) of the patch. Furthermore, because pathogens compete for resources (patches) with other pathogens, epidemiological models are useful in addressing questions of coexistence and stability of biological systems.

Patch dynamics usually takes place on a different time scale than the pathogen's dynamics. Furthermore, usually natural selection acts faster on the evolution of a pathogen than on the evolution of the host. At this time, there is no clear mathematical framework in which these questions can be properly addressed. However, epidemiological models have provided a useful framework to begin to study these evolutionary dynamics (see Levin 1983a, b; May and Anderson 1983a, b; Levin and Castillo-Chavez 1989; Anderson and May 1991;). Specific coevolutionary interactions such as those 
experienced by the myxoma virus (fairly lethal) and the European rabbit have provided an excellent and challenging opportunity for the application of epidemiological models to questions in evolutionary biology (see Levin and Pimentel 1981; Levin 1983a, b; May and Anderson 1983a, b).

The goals of this chapter are limited. We provide a brief introduction to epidemiological models while, in the process, we try to provide an introduction to epidemiological "thinking" (e.g. we discuss the thought process that goes or that we think goes into building models that address specific epidemiological questions). Our introduction to epidemiological "thinking" is handled through the insertion of remarks and comments throughout the text. Many remarks are, in fact, obvious but nevertheless (we think) important. The prior neglect of some of the ideas inserted in these remarks was inspired by mathematical convenience. Unfortunately, it kept mathematical epidemiology away from the field of theoretical epidemiology a situation that has begun to change (see the recent encyclopedic volume of Anderson and May 1991). Section 2 of this chapter introduces the basic epidemiological models and we refer to Section 2 as the section on Alphabet Models because we follow the useful mnemonic description introduced by Hethcote (1976). The arrival of the HIV/AIDS epidemic reactivated the study of the effects of long and variable periods of infection on disease dynamics. Section 3 illustrates the incorporation of variable waiting and transition times into epidemiological models. A more general approach would include age-of-infection models (see Thieme and Castillo-Chavez 1989, 1993) which would require a longer and more elaborate chapter, and it is therefore omitted. Section 4 adds social structure in the context of population dynamics. We focus on the role of social structure in demographic processes (pair-formation and dissolution) because their extension to epidemiological processes (more or less straightforward) would lengthen this chapter. Section 5 provides an outline of how these ideas are incorporated into the stochastic world. We discuss these extensions in the realm of continuous time Markov chain processes while borrowing the language of percolation models (see Luo and Castillo-Chavez 1991; Castillo-Chavez et al. 1994a). Sections 4 and 5 are less detailed than prior sections because of space limitations. However, we provide a large set of references to help the interested reader fill in the gaps. Section 6 summarizes the contents of this chapter with a few closing thoughts.

\section{Alphabet Models}

Alphabet models (see Hethcote, 1976) consider a population of susceptible individuals which is being invaded by an infectious agent. These models concentrate on the disease dynamics that result if a small number of members of the population become infected and (eventually) infectious. The population is usually divided into three epidemiological subclasses: labeled $S, I$, and $R$. $S(t)$ denotes those individuals who are susceptible to the disease at time $t, I(t)$ denotes the number of infected individuals (here assumed infectious and hence capable of spreading the disease) at time $t$, and $R(t)$ denotes the number of individuals who at time $t$ no longer contribute to the direct spread of the 
disease because they have been removed through isolation or immunization, or through recovery from the disease with immunity against re-infection, or through death caused by the disease. The characterizations of $R(t)$ are very different from an epidemiological point of view, however, they are equivalent in a modeling sense because these individuals do not contribute further to the spread of disease and because the models do not incorporate demographic effects. For models that also involve demographic effects the situation can be very different. The total population involved in the disease dynamics at time $t$ will be denoted by $N(t)$ where $N(t)$ may be equal to $S(t)+I(t)$ or $S(t)+I(t)+$ $\mathrm{R}(\mathrm{t})$.

A model was proposed by Kermack and MacKendrick in 1927 to explain the rapid rise and fall of cases observed frequently in epidemics such as the Great Plague in London (1665-1666), the cholera epidemic in London (1865) and the plague in Bombay (1906). The simplest version of their model with a trivial modification is given by the following set of nonlinear ordinary differential equations (ODE's):

$$
\begin{gathered}
\mathrm{S}^{\prime}=-\beta \mathrm{S} \mathrm{I} / \mathrm{N}, \\
\mathrm{I}^{\prime}=\beta \mathrm{SI} / \mathrm{N}-\frac{1}{\tau} \mathrm{I}, \\
\mathrm{R}^{\prime}=\frac{1}{\tau} \mathrm{I},
\end{gathered}
$$

where (usually) $N(t)=S(t)+I(t)+R(t)$.

This model is based on the following three assumptions:

(i) There are no deaths unrelated to the disease and no births. This assumption is reflected in the relation $(S+I+R)^{\prime}=0$, so that total population size $N(t)$ is constant. It is a reasonable assumption for epidemics of short duration. [If there are disease-related deaths or if $R$ is used to label individuals that are permanently removed from the population and, hence, unable to interact with S- and I-individuals, then a better definition of $\mathrm{N}(\mathrm{t})$ would be $\mathrm{N}(\mathrm{t}) \equiv \mathrm{S}(\mathrm{t})+\mathrm{I}(\mathrm{t})]$.

(ii) The homogeneous mixing assumption, that is, the probability that a susceptible has a contact with an infective is $I / N$. The contact process for spreading the infection is based on contacts between infectives and susceptibles and these contacts occur at random (homogeneous mixing). Here $\beta \equiv \beta(\mathrm{N})$ denotes the per capita effective contact rate which in general is a function of the size of the active population. It is common to assume that $\beta(\mathrm{N})=\mathrm{p} \phi(\mathrm{N})$ where $\mathrm{p}$ denotes the probability of transmission per contact and $\phi(\mathrm{N})$ denotes the per capita contact rate. Hence $\phi S$ gives the average number of contacts that the population of susceptibles experience per unit time. These susceptibles have contacts with S-, I-, and R-individuals but under the homogeneous mixing assumption the proportion of contacts with infectives is $\mathrm{I} / \mathrm{N}$. The total average contact rate (handshakes, kisses, etc.) is 
$\phi \mathrm{SI} / \mathrm{N}$, but not all contacts lead to infection. The effective contact rate or the incidence (new cases of infection per unit of time) is $\beta(\mathrm{N}) \mathrm{SI} / \mathrm{N}$. [If $\mathrm{N}$ is constant, then the rate of new infections is proportional to both the number of susceptibles and infectives; this is also the case if $\mathrm{N}$ is not constant but $\beta(\mathrm{N}) \equiv \mathrm{pN}$--a linear function of $\mathrm{N}$. The use of a bilinear incidence rate corresponds to the use of the the mass-action law].

(iii) The per capita recovery rate is constant, that is, infectives are removed from the I-class into the $R$-class at the constant rate $\frac{1}{\tau}$ (per individual and per unit of time). [This assumption implies that the distribution of time spent by individuals in the I-class has an exponential distribution with mean $\tau$ ].

A key question is whether or not a population experiences an epidemic, that is, whether or not a pathogen can successfully invade a population. Success here means that the number of patches occupied, that is, the number of infective individuals, increases temporarily over time after the initial introduction of a pathogen into a population of purely susceptible patches. Under some conditions, the pathogen succeeds and there is an epidemic (the number of infected patches rises); otherwise it fails. These conditions are usually formulated in terms of a threshold value: the basic reproductive number or the contact number. If this value exceeds a critical value (usually one) there is an epidemic. Otherwise there is no epidemic (for a mathematical definition of this concept that is well grounded in the biology the reader is referred to Diekmann et al. (1990).

From the above model, Kermack and MacKendrick (1927) deduced their threshold theorem. The importance of their result is due in part to the fact that most epidemic models exhibit similar threshold phenomena. Kermack and MacKendrick found a dimensionless quantity called the contact, or the basic reproductive number, whose magnitude determines whether the infection will die out (I decreases monotonically to zero) or there will be an epidemic (I first increases to a maximum and then decreases to zero).

Since $S+I+R$ is constant, we may either let $S+I+R=K$, the constant total population size, or we may measure $S$, I, and $R$ in units of $K$, effectively dividing $S$, $I$, and $R$ by $K$ so that they now represent fractions of the population in each class with $S+I+R=1$. In either case, we may eliminate $R$ from the system by substituting $R=K-S-I$ or $R=1-S-I$. We choose to work with the original variables and obtain the two-dimensional system:

$$
\begin{gathered}
\mathrm{S}^{\prime}=-\beta \mathrm{SI} / \mathrm{K}, \\
\mathrm{I}^{\prime}=\beta \mathrm{SI} / \mathrm{K}-\frac{1}{\tau} \mathrm{I} .
\end{gathered}
$$

Here $\beta$ is measured in units of contacts per unit time and $\tau$ is measured in units of time. 
We observe that $S^{\prime}<0$ for all $t$ and $I^{\prime}<0$ if and only if $S<\frac{K}{\beta \tau}$. This implies that if $\mathrm{S}(0)<\frac{\mathrm{K}}{\beta \tau}$, then $\mathrm{I}^{\prime}<0$ for all $\mathrm{t}$ and the infection dies out, but if $\mathrm{S}(0)>\frac{\mathrm{K}}{\beta \tau}$, then $\mathrm{I}^{\prime}>0$ while $\mathrm{S}$ decreases to $\frac{\mathrm{K}}{\beta \tau}$, after which $\mathrm{I}^{\prime}<0$. If we "think" of introducing a small number of infectives into a susceptible population at time zero, so that $\mathrm{I}(0)=\epsilon>0, \mathrm{R}(0)=0, \mathrm{~S}(0)=\mathrm{K}-\epsilon \approx \mathrm{K}$. then we have a threshold dimensionless quantity

$$
\mathscr{\Re}_{0}=\beta \tau \text {. }
$$

If $\mathscr{R}_{0}<1$ the infection dies out and if $\mathscr{R}_{0}>1$ there is an epidemic. This is the threshold theorem of Kermack and MacKendrick. [Kermack and MacKendrick used the mass-action law, that is, they implicitly assumed that $\beta \equiv \mathrm{pK}$. To simplify the notation we assume without loss of generality that $\mathrm{K}=1$ during the mathematical analysis of the Kermack and MacKendrick model. [Note that this assumption does not alter the value of $\mathscr{R}_{0}$ as $\beta$ is assumed to be a constant; however, it does make a quantitative difference if we assume, as Kermack and MacKendrick probably did that $\beta \equiv \mathrm{pK}$, in which case $\beta$ would have to be measured in different units].

A qualitative analysis of the system of differential equations (here we take $K=1$ to simplify the analysis) shows that equilibria are solutions $\left(\mathrm{S}_{\infty}, \mathrm{I}_{\infty}\right)$ of $\mathrm{S}_{\infty} \mathrm{I}_{\infty}=0, \mathrm{I}_{\infty}\left(\beta \mathrm{S}_{\infty}-\frac{1}{\tau}\right)=0$. Thus there is a line of equilibria $\left(S_{\infty}, 0\right)$ with arbitrary $S_{\infty}, 0 \leq S_{\infty} \leq 1$. The linearization at an equilibrium $\left(\mathrm{S}_{\infty}, 0\right)$ is

$$
\begin{gathered}
\mathbf{u}^{\prime}=-\beta \mathrm{S}_{\infty} \mathbf{v} \\
\mathbf{v}^{\prime}=\left(\beta \mathrm{S}_{\infty}-\frac{1}{\tau}\right) \mathbf{v}
\end{gathered}
$$

with matrix

$$
\left[\begin{array}{cc}
0 & -\beta S_{\infty} \\
0 & \beta S_{\infty}-\frac{1}{\tau}
\end{array}\right]
$$

having eigenvalues 0 and $\beta \mathrm{S}_{\infty}-\frac{1}{\tau}$. The zero eigenvalue means that linearization is useless for the direct mathematical analysis of the system. However, it is not difficult to show that the phase portrait is as in Figure 1.

Hence we observe, that the limiting value $S(\infty)$ of each orbit depends on the starting point $S(0)$, but 
$S(\infty)>0$ for all $S(0)$. This means that in an epidemic not all members of the population become infected, a deduction that agrees with recorded data for many epidemics. It is not difficult to show that for an orbit which goes from the point $(S(0), 0)$ to the point $(S(\infty), 0)$,

$$
S(\infty)-\frac{1}{\beta \tau} \ln S(\infty)=S(0)-\frac{1}{\beta \tau} \ln S(0)
$$

To see this, we observe that

$$
\begin{aligned}
\frac{\mathrm{d}}{\mathrm{dt}}\left[\mathrm{S}+\mathrm{I}-\frac{1}{\beta \tau} \ln \mathrm{S}\right] & =\mathrm{S}^{\prime}+\mathrm{I}^{\prime}-\frac{1}{\beta \tau} \frac{\mathrm{S}^{\prime}}{\mathrm{S}} \\
& =\mathrm{S}^{\prime}\left[1-\frac{1}{\beta \tau \mathrm{S}}\right]+\mathrm{I}^{\prime} \\
& =-\beta \mathrm{SI}\left[1-\frac{1}{\beta \tau \mathrm{S}}\right]+\mathrm{I}^{\prime} \\
& =-\beta \mathrm{SI}+\frac{1}{\tau} \mathrm{I}+\mathrm{I}^{\prime}=0 .
\end{aligned}
$$

Thus $\mathrm{S}(\mathrm{t})+\mathrm{I}(\mathrm{t})-\frac{1}{\beta \tau} \ln \mathrm{S}(\mathrm{t})$ is a constant and

$$
\mathrm{S}(\infty)+\mathrm{I}(\infty)-\frac{1}{\beta \tau} \ln \mathrm{S}(\infty)=\mathrm{S}(0)+\mathrm{I}(0)-\frac{1}{\beta \tau} \ln \mathrm{S}(0)
$$

Since $I(\infty)=I(0)=0$ it follows that

$$
\mathrm{S}(\infty)-\frac{1}{\beta \tau} \ln \mathrm{S}(\infty)=\mathrm{S}(0)-\frac{1}{\beta \tau} \ln \mathrm{S}(0)
$$

The contact rate $\beta$ in the model we are considering (assumed constant), depends on the particular disease being studied, and may also depend on social and behavioral factors. In general, it is difficult to estimate $\beta$, or equivalently $\mathscr{F}_{0}$ (since $\tau$ can be estimated experimentally). However, as $\mathrm{S}(0) \sim \mathrm{K}$ as a disease is invading a purely susceptible population and $S(\infty)$, that is, the proportion of individuals with antibodies, can be estimated from serological data. The above relation gives a means of calculating $\beta \tau$ for a given epidemic from knowledge of $\mathrm{S}(0)$ and $\mathrm{S}(\infty)$. For example, an influenza epidemic has been reported with $S(0)=0.911, S(\infty)=0.513$. From

we derive

$$
S(0)-S(\infty)=\frac{1}{\beta \tau}[\ln S(0)-\ln S(\infty)]=\frac{1}{\beta \tau} \ln \frac{S(0)}{S(\infty)}
$$

$$
\beta \tau=\frac{\ln \frac{S(0)}{S(\infty)}}{S(0)-S(\infty)}
$$


and the given data yields $\beta \tau=1.44$, and since $\tau$ is about 3 days then $\beta \sim 0.48 /$ day. Note that as long as $\mathrm{S}(0)>\frac{1}{\beta \tau}$, that is, as long as the proportion of susceptibles exceeds a critical value, an epidemic is possible (the data is from Evans 1982 as reported in Hethcote 1989).

The Kermack-MacKendrick model considered above is an example of a simple model in which the population is divided into different compartments and the transitions between compartments are modeled. The understanding derived from simple models is useful in describing general properties and outlining the possible effects of control programs. It is also possible to formulate complex models with detailed assumptions incorporating more structure appropriate to a particular disease or situation. For example, compartments may be subdivided further to describe situations in which different subclasses have different transmission rates corresponding to different behavior (as in sexually transmitted diseases), age dependence, seasonal variations, or spatial dependence. Such more complex models may give more specific quantitative predictions.

The Kermack-MacKendrick model is described as an SIR model because the transitions are from susceptible to infective to removed, with the removal coming through recovery with full immunity (as in measles) or through death (as in rabies and many other animal diseases). Another type of model is an SIS model, in which infectives return to the susceptible class on recovery because the disease confers no immunity. Such models are appropriate for most diseases transmitted by bacterial or helminth agents, and for some sexually transmitted diseases including gonorrhea but not for others like HIV or herpes that infect individuals for life.

The simplest SIS model, also due to Kermack and MacKendrick (1932), is

$$
\begin{aligned}
\mathrm{S}^{\prime} & =-\beta \mathrm{S} \mathrm{I} / \mathrm{N}+\frac{1}{\tau} \mathrm{I} \\
\mathrm{I}^{\prime} & =\beta \mathrm{S} \mathrm{I} / \mathrm{N}-\frac{1}{\tau} \mathrm{I}
\end{aligned}
$$

where $\mathrm{S}+\mathrm{I}=\mathrm{N}$.

The SIS model differs from the SIR model because recovered members return to the class $S$ at a rate $\frac{1}{\tau} \mathrm{I}$ instead of passing to the class $R$. Again, the total population size is a constant $N=K$, and we may measure it in units of $K$ (that is, we can assume that $K=1$ ). We may use the relation $I$ $=\mathrm{K}-\mathrm{S}$ to reduce the model to the single equation

$$
\mathrm{S}^{\prime}=(1-\mathrm{S} / \mathrm{K})\left(-\beta \mathrm{S}+\frac{\mathrm{K}}{\tau}\right)
$$

with two equilibria,

$$
S=K \text { and } S=\frac{K}{\beta \tau} .
$$


If $\frac{1}{\beta \tau}<\frac{\mathrm{S}}{\mathrm{K}}$, or $\beta \tau>1$ both these equilibria are in the interval $0<\mathrm{S} \leq \mathrm{K}$ but if $\frac{1}{\beta \tau}>\frac{\mathrm{S}}{\mathrm{K}}$ or $\beta \tau<1$ only the equilibrium $\mathrm{S}=\mathrm{K}$ is relevant. The threshold quantity $\beta \tau$ again distinguishes between two qualitatively different possible behaviors, but the possibilities are different from the SIR model. To simplify the analysis we now $K=1$. Note that our modification of the incidence rate and the fact that $\beta$ is a constant implies that $K$ plays no role in the discussion.

The linearization about the equilibrium $\mathrm{S}=1$ ( or $\mathrm{I}=0$ ), obtained with the aid of the change of variable $u=1-S$, is

$$
\mathbf{u}^{\prime}=\left(\beta-\frac{1}{\tau}\right) \mathbf{u}
$$

Thus the equilibrium is asymptotically stable if $\beta-\frac{1}{\tau}<0$, or $\beta \tau<1$, and unstable if $\beta \tau>1$. The linearization about the equilibrium $S=\frac{1}{\beta \tau},(\mathrm{K}=1)$ is of interest only if $\beta \tau>1$, and it is obtained through the change of variable $u=S-\frac{1}{\beta \tau}$. It is

$$
\mathrm{u}^{\prime}=\left(\frac{1}{\tau}-\beta\right) \mathrm{u},
$$

and thus the equilibrium is asymptotically stable if $\frac{1}{\tau}-\beta<0$, or $\beta \tau>1$. The result of this analysis is that there is always a single asymptotically stable equilibrium to which all solutions tend. If $\beta \tau<1$, this equilibrium is $\mathrm{S}=1, \mathrm{I}=0$, corresponding to the disappearance of the disease. If $\beta \tau>1$, the asymptotically stable equilibrium is $\mathrm{S}=\frac{1}{\beta \tau}, \mathrm{I}=1-\frac{1}{\beta \tau}>0$, called an endemic equilibrium, in which the disease persists.

Measles is a disease for which endemic equilibria have been observed in many places, with oscillations about the equilibrium. In an attempt to formulate an SIR model which could give such behavior, Soper proposed a model in 1929 which assumed a constant birth rate $\mu \mathrm{K}$ in the susceptible class and a constant death rate $\mu \mathrm{K}$ in the removed class,

$$
\begin{gathered}
\mathrm{S}^{\prime}=-\frac{\beta \mathrm{S}}{\mathrm{K}} \mathrm{I}+\mu \mathrm{K} \\
\mathrm{I}^{\prime}=\frac{\beta \mathrm{S}}{\mathrm{K}} \mathrm{I}-\frac{1}{\tau} \mathrm{I} \\
\mathrm{R}^{\prime}=\frac{1}{\tau} \mathrm{I}-\mu \mathrm{K} .
\end{gathered}
$$

This model is unsatisfactory because the linkage of births of susceptibles to deaths of removed members is unreasonable. It is also an improper model mathematically because if $R(0)$ and $I(0)$ are sufficiently 
small then $R(t)$ will become negative. Furthermore, as discussed before, this model implicitly assumes that $\beta$ is a function of $\mathrm{K}$ and hence one must be careful with the units. For any disease model to be plausible, it is essential that the problem be properly posed in the sense that the number of members in each class must remain non-negative. [A full analysis of a model should include a verification of this property].

The difficulties in Soper's model were resolved by the assumption of deaths in each class proportional to the number of members in the class, keeping the total population size constant (Hethcote 1976)

$$
\begin{gathered}
\mathrm{S}^{\prime}=-\beta \mathrm{SI} / \mathrm{K}+\frac{1}{\mathrm{~L}}(\mathrm{~K}-\mathrm{S}) \\
\mathrm{I}^{\prime}=\beta \mathrm{SI} / \mathrm{K}-\frac{1}{\tau} \mathrm{I}-\frac{1}{\mathrm{~L}} \mathrm{I} \\
\mathrm{R}^{\prime}=\frac{1}{\tau} \mathrm{I}-\frac{1}{\mathrm{~L}} \mathrm{R} .
\end{gathered}
$$

Here $\mathrm{L}$ represents the average life span (to be justified later) because $1 / \mathrm{L}$ is the death rate.

Because $\mathrm{S}+\mathrm{I}+\mathrm{R}=\mathrm{K}$, we can eliminate $\mathrm{R}$ and consider the two-dimensional system

$$
\begin{aligned}
\mathrm{S}^{\prime} & =-\beta \mathrm{S} \mathrm{I} / \mathrm{K}+\frac{1}{\mathrm{~L}}(\mathrm{~K}-\mathrm{S}) \\
\mathrm{I}^{\prime} & =\beta \mathrm{S} \mathrm{I} / \mathrm{K}-\frac{1}{\tau} \mathrm{I}-\frac{1}{\mathrm{~L}} \mathrm{I} .
\end{aligned}
$$

From the equilibrium conditions $\beta \mathrm{S} \mathrm{I} / \mathrm{K}=\frac{1}{\mathrm{~L}}(\mathrm{~K}-\mathrm{S})$ and $\mathrm{I}\left(\beta \mathrm{S} / \mathrm{K}-\frac{1}{\tau}-\frac{1}{\mathrm{~L}}\right)=0$, we see that there is always an equilibrium $S=K, I=0$ (vanishing of the disease), and if $I^{\prime}>0$, that is, if

$$
\frac{\tau+\mathrm{L}}{\beta \tau \mathrm{L}}<\frac{\mathrm{K}}{\mathrm{S}(0)}
$$

or (if everybody is susceptible, that is, if $\mathrm{S}(0) \approx \mathrm{K}$ ) if

$$
\beta \tau\left(\frac{\mathrm{L}}{\mathrm{L}+\tau}\right)>1
$$

there is a second equilibrium $\mathrm{S}=\frac{\tau+\mathrm{L}}{\beta \tau \mathrm{L}} \mathrm{K}$ with $\mathrm{I}>0$ (endemic equilibrium). Using linearization, it is not difficult to show that if

$$
\beta \tau\left(\frac{\mathrm{L}}{\mathrm{L}+\tau}\right)<1
$$

the equilibrium $\mathrm{S}=\mathrm{K}, \mathrm{I}=0$ is asymptotically stable, while if

$$
\beta \tau\left(\frac{\mathrm{L}}{\mathrm{L}+\tau}\right)>1
$$

then is unstable.

The threshold

$$
\mathscr{R}_{0}=\beta \tau\left(\frac{\mathrm{L}}{\mathrm{L}+\tau}\right)
$$


is the analogous of the Kermack-MacKendrick model except that $\tau$ needs to be corrected to take into account the effects of natural mortality on the length of the infectious period, that is,

$$
\tau\left(\frac{\mathrm{L}}{\mathrm{L}+\tau}\right)
$$

denotes the death-adjusted mean infectious period.

The equilibrium $\mathrm{S}=\mathrm{K}, \mathrm{I}=0$ is unstable and the endemic equilibrium is asymptotically stable if $\mathscr{B}_{0}>1$. Thus the model allows endemic equilibria. In conclusion, the SIR model with births and deaths and the SIS model both support endemic equilibria, and hence, we may also conclude that the requirement for the support of an endemic equilibrium is the flow of new susceptibles, either through births or through recovery without immunity.

In general, if $\beta(\mathrm{N}) \equiv \mathrm{p} \phi(\mathrm{N})$ then the condition $\mathscr{R}_{0}>1$ is equivalent to

$$
\phi(\mathrm{S}(0))>\frac{\tau+\mathrm{L}}{\mathrm{p} \tau \mathrm{L}}
$$

or if $\phi$ has an inverse, to the condition

$$
\mathrm{S}(0)>\mathrm{N}_{\text {critical }} \equiv \phi^{-1}\left(\frac{\tau+\mathrm{L}}{\mathrm{p} \tau \mathrm{L}}\right)
$$

that is, the susceptible population must reach a critical value for an epidemic to occur. This is a useful concept; however the estimation of the critical value of $\mathrm{N}$ depends on our knowledge of the functional form of $\beta(N)$. Rough values of $N_{\text {critical }}$ have been estimated implicitly assuming that $\beta(\mathrm{N})=\mathrm{pN}$, that is, $\mathrm{N}_{\text {critical }}$ has been computed (indirectly from data) using the formula

$$
\mathrm{N}_{\text {critical }} \equiv \frac{\tau+\mathrm{L}}{\mathrm{p} \tau \mathrm{L}}
$$

These estimates have led to the conclusion that influenza will persist in continental cities with at least 300,000 individuals but it will only persist in islands with at least 500,000 individuals (for explicit details see Anderson, 1982). The values are different because the p's are probably different as well as the average age of first infection. An alternative way of reading these results (using available data) is as follows: if $\mathrm{N}$ is at least 300,000 (for cities) then $\mathrm{p}$ (the probability of transmission per contact) is high enough to have an infection that will remain endemic. The same result holds for islands as long as $\mathrm{N}$ is at least 500,000 . To summarize these results to make the data consistent with the same functional form for $\beta(\mathrm{N})$ we must accept different values of $\mathrm{p}$ for islands and continental cities.

Thresholds are useful as they help us determine approaches for disease eradication or control. In order to eradicate an infection, it is necessary to reduce the contact number $\mathscr{R}_{0}$ below 1 . This may 
sometimes be achieved by immunization, which has the effect of transferring members from the susceptible class to the removed class and thus reducing $K$ (here $K=1$ ). The effect of immunizing a fraction (which could be a function) $q$ of the population is to replace $K$ by $K(1-q)$, and thus to replace the contact number $\mathscr{R}_{0}=\beta \tau\left(\frac{\mathrm{L}}{\mathrm{L}+\tau}\right)$ by $\beta \tau\left(\frac{\mathrm{L}}{\mathrm{L}+\tau}\right)(1-\mathrm{q})$. The condition

$$
\beta \tau\left(\frac{\mathrm{L}}{\mathrm{L}+\tau}\right)(1-\mathrm{q})<1 \text { gives } 1-\mathrm{q}=\frac{1}{\mathrm{R}_{0}} \text {, or } \mathrm{q}>1-\frac{1}{\mathscr{R}_{0}}
$$

guarantees that success has been achieved

A population is said to have herd immunity if a large enough fraction has been immunized to assure that the disease will not spread if an infective case is introduced. The only infection for which this has actually been achieved worldwide is smallpox (there are still 600 laboratory samples in Russia and the USA that were supposed to be destroyed by the end of 1993). That is, through vaccination we will have eliminated a species (we have just learned that they were not destroyed and that an extension has been obtained). For measles, epidemiological data in the U.S. indicates that $\mathscr{R}_{0}$ for rural populations (using models for which $\beta=\mathrm{pN}$, see later sections) ranges from 5.4 to 6.3 , requiring vaccination of $81.5 \%$ to $84.1 \%$ of the population. In urban areas, $\mathscr{R}_{0}$ ranges from 8.3 to 13.0 , requiring vaccination of $88.0 \%$ to $92.3 \%$ of the population. In Great Britain, $\mathscr{B}_{0}$ ranges from 12.5 to 16.3 , requiring vaccination of $92 \%-94 \%$ of the population. As vaccine efficacy for measles vaccination at age 15 months is approximately $95 \%$, it is effectively impossible to achieve herd immunity for measles (this information is taken from Anderson 1982). Smallpox has a lower value of $R_{0}$ and requires about $80 \%$ immunization. As the consequences of the disease are more serious, this is achievable. [The situation is not as simple as the above models assume homogeneous mixing. In fact, the difficulties experienced in eradicating smallpox were due to heterogeneity in the contact rates, for an earlier paper on immunization see Hethcote (1978) and references therein. Only after an intensive campaign that took into account these heterogeneities was it possible to eradicate smallpox.] The homogeneous mixing model only says that it is feasible. It also says that it is nearly impossible to eradicate measles.

The SIR model with births and deaths predicts part of what is observed for measles but does not support the possibility of sustained oscillations about the endemic equilibrium. To explain oscillations about the endemic equilibrium as observed, we might assume seasonal variations in the contact rate $\beta$ - not an unreasonable supposition for a childhood disease most commonly transmitted through school contacts, especially in winter in cold climates.

The SIR model with births and deaths is quite inappropriate for a disease such as rabies from which recovery is rare. For such a disease, the class $R$ consists of members removed by death, and the total population size is $\mathrm{S}+\mathrm{I}$. Thus the number of births should be proportional to $(\mathrm{S}+\mathrm{I})$, or possibly proportional to $\mathrm{S}$ to reflect the fact that such diseases are sufficiently debilitating that 
infective members do not reproduce. It is not possible to assume this and also keep the total population size constant in the absence of disease. A plausible model for such a disease will necessarily assume a nonlinear birth rate and will imply that the total population size must vary in time whether or not the disease is present. The formulation and mathematical analysis of general models that include these features can be found in the works of Brauer $(1990,1991)$ and Pugliese $(1990 \mathrm{a}, 1990 \mathrm{~b})$.

\section{Transition Rates and Waiting Times}

In the models we have been considering, we have assumed rates of transition between classes which are proportional to the size of the class, such as recovery rates $\frac{1}{\tau} \mathbf{I}$. Such assumptions do not really mean that recovery depends on the size of the infective class. A more realistic formulation is obtained if we replace the differential equation

$$
\mathrm{I}^{\prime}=\beta \mathrm{SI} / \mathrm{N}-\frac{1}{\tau} \mathrm{I}
$$

by the integral equation

$$
\mathrm{I}(\mathrm{t})=\int_{0}^{\mathrm{t}} \beta \mathrm{S}(\mathrm{t})[\mathrm{I}(\mathrm{x}) / \mathrm{N}(\mathrm{x})] \mathrm{e}^{-\frac{1}{\tau}(\mathrm{t}-\mathrm{x})} \mathrm{d} \mathrm{x}-.
$$

If $t$ is large enough for members who were initially (at time $t=0$ ) infective to have recovered and we assume that $\mathrm{N}(\mathrm{x})=\mathrm{K}$, a constant, then this integral equation is equivalent to the differential equation because differentiation under the integral sign gives

$$
\begin{aligned}
\mathrm{I}^{\prime}(\mathrm{t}) & =\beta \mathrm{S}(\mathrm{t}) \mathrm{I}(\mathrm{t}) / \mathrm{K}+\int_{0}^{\mathrm{t}} \beta \mathrm{S}(\mathrm{x})[\mathrm{I}(\mathrm{x}) / \mathrm{K}] \frac{\mathrm{d}}{\mathrm{dt}} \mathrm{e}^{-\frac{1}{\tau}(\mathrm{t}-\mathrm{x})} \mathrm{dx} \\
& =\beta \mathrm{S}(\mathrm{t}) \mathrm{I}(\mathrm{t}) / \mathrm{K}-\frac{1}{\tau} \int_{0}^{\mathrm{t}} \beta \mathrm{S}(\mathrm{x})[\mathrm{I}(\mathrm{x}) / \mathrm{K}] \mathrm{e}^{-\frac{1}{\tau}(\mathrm{t}-\mathrm{x})} \mathrm{d} \mathrm{x} \\
& =\beta \mathrm{S}(\mathrm{t}) \mathrm{I}(\mathrm{t}) / \mathrm{K}-\frac{1}{\tau} \mathrm{I}(\mathrm{t}) .
\end{aligned}
$$

The interpretation of the integral equation is that $\mathrm{e}^{-\frac{1}{\tau} \mathrm{s}}$ is the probability of remaining infective at time $\mathrm{s}$ after having become infective. Then

$$
\beta \mathrm{S}(\mathrm{x})[\mathrm{I}(\mathrm{x}) / \mathrm{K}] \mathrm{e}^{-\frac{1}{\tau}(\mathrm{t}-\mathrm{x})}
$$

represents the number of members who became infective at time $x$ and remain infective $(t-x)$ time units later, and

$$
\int_{0}^{\mathrm{t}} \beta \mathrm{S}(\mathrm{x})[\mathrm{I}(\mathrm{x}) / \mathrm{K}] \mathrm{e}^{-\frac{1}{\tau}(\mathrm{t}-\mathrm{x})} \mathrm{dx}
$$

is the integral over all times $\mathrm{x} \leq \mathrm{t}$ of this number or the total number of infectives at time $\mathrm{t}$. The 
mean length of the infective period is

$$
\int_{0}^{\infty} \mathrm{e}^{-\frac{1}{\tau} \mathrm{s}} \mathrm{ds}=\tau .
$$

A similar interpretation applies to the constant of proportionality $\frac{1}{\mathrm{~L}}$ in the death rate in each class in the $\mathrm{S}-\mathrm{I}-\mathrm{R}$ model with births and deaths. The reinterpretation is that

$$
e^{-\frac{1}{L} s}
$$

is the probability of survival to age $\mathrm{s}$, and thus the average life expectancy is $\mathrm{L}$.

We can also give a waiting-time interpretation to the rate of infection in the SIR model with births and deaths. At the endemic equilibrium $\left(S_{\infty}, I_{\infty}\right)$ the rate of new infections is $\beta \mathrm{S}_{\infty}\left[\mathrm{I}_{\infty} / \mathrm{K}\right]$. This means that $\beta\left[\mathrm{I}_{\infty} / \mathrm{K}\right]$ is the rate of infection per susceptible. If we assume an exponential distribution of probability of infection of an individual, the average time spent in the susceptible class before infection is $\frac{\mathrm{K}}{\beta \mathrm{I}_{\infty}}$. Thus the average age at infection, which we denote by $\mathrm{A}$, is $\frac{\mathrm{K}}{\beta \mathrm{I}_{\infty}}$. As we have seen for this model, the endemic equilibrium $\left(\mathrm{S}_{\infty}, \mathrm{I}_{\infty}\right)$ is the solution with $\mathrm{I}_{\infty}>0$ of the pair of equations

$$
\beta \mathrm{S}_{\infty}\left[\mathrm{I}_{\infty} / \mathrm{K}\right]=\frac{1}{\mathrm{~L}}\left(\mathrm{~K}-\mathrm{S}_{\infty}\right),\left[\mathrm{I}_{\infty} / \mathrm{K}\right]\left[\beta \mathrm{S}_{\infty}-\frac{1}{\tau}-\frac{1}{\mathrm{~L}}\right]=0
$$

from which we obtain

FF

$$
\mathrm{S}_{\infty}=\frac{\tau+\mathrm{L}}{\beta \tau \mathrm{L}} \mathrm{K}, \mathrm{I}_{\infty}=\frac{\mathrm{K}}{\beta \mathrm{L} \mathrm{S}_{\infty}}\left(\mathrm{K}-\mathrm{S}_{\infty}\right)
$$

Also, the contact number is

$$
\mathscr{R}_{0}=\beta \tau \frac{\mathrm{L}}{\tau+\mathrm{L}}=\frac{\mathrm{K}}{\mathrm{S}_{\infty}}
$$

with $\mathscr{R}_{0}>1$. From these relations, we see that

The relation

$$
\frac{\mathrm{L}}{\mathrm{A}}=\beta \mathrm{L}\left[\mathrm{I}_{\infty} / \mathrm{K}\right]=\frac{\mathrm{K}}{\mathrm{S}_{\infty}}\left(1-\left[\mathrm{S}_{\infty} / \mathrm{K}\right]\right)=\frac{\mathrm{K}}{\mathrm{S}_{\infty}}-1=\mathscr{R}_{0}-1
$$

$$
\Re_{0}=1+\frac{L}{A}
$$

is a useful means of estimating contact numbers since $\mathrm{L}$ and $\mathrm{A}$ can be measured. Note that this 
derivation assumes the existence of an endemic state and, consequently that $\mathscr{B}_{0}>1$. The estimates given earlier for contact numbers in various measles outbreaks were derived from this relation. One of the effects of immunization programs is to reduce the number of infectives at equilibrium, and this means that the average age of infection will increase. This could have serious consequences in childhood diseases for which the danger of complications increases with age. However, immunization programs will tend to reduce the total number of cases in older individuals even though they tend to increase the proportion of older cases.

Instead of assuming exponentially distributed recovery rates, we could assume that there is a probability $\mathrm{P}(\mathrm{s})$ of remaining infective a time $\mathrm{s}$ after becoming infective, where $\mathrm{P}(\mathrm{s})$ is a monotone decreasing function with $\mathrm{P}(0)=1$. We will let $\infty$

$$
\tau=\int_{0}^{\infty} \mathrm{P}(\mathrm{s}) \mathrm{ds}
$$

the average length of the infective period. An important special case is the choice

$$
\mathrm{P}(\mathrm{s})= \begin{cases}1 & , 0 \leq \mathrm{s} \leq \tau \\ 0 & , \mathrm{~s}>\tau\end{cases}
$$

corresponding to an infective period of fixed length $\tau$ In this case, the integral equation for $\mathrm{I}(\mathrm{t})$, namely

$$
\mathrm{I}(\mathrm{t})=\int_{0}^{\mathrm{t}} \beta \mathrm{S}(\mathrm{x}) \mathrm{I}(\mathrm{x}) \mathrm{P}(\mathrm{t}-\mathrm{x}) \mathrm{d} \mathrm{x}
$$

becomes

$$
I(t)=\int_{t-\tau}^{t} \beta S(x) I(x) d x
$$

which is equivalent to the differential-difference equation

$$
\mathrm{I}^{\prime}(\mathrm{t})=\beta \mathrm{S}(\mathrm{t}) \mathrm{I}(\mathrm{t})-\beta \mathrm{S}(\mathrm{t}-\tau) \mathrm{I}(\mathrm{t}-\tau)
$$

The

choices $\mathrm{P}(\mathrm{s})=\mathrm{e}^{-\frac{1}{\tau} \mathrm{s}}$ and $\mathrm{P}(\mathrm{s})=\left\{\begin{array}{ll}1 & , 0 \leq \mathrm{s} \leq \tau \\ 0 & , \mathrm{~s}>\tau\end{array} \quad\right.$ represent extremes,

and there is some reason to conjecture that models with different choices of the distribution function $\mathrm{P}(\mathrm{s})$ with the same average infective period $\tau$ should have the same qualitative behavior. Whether this is true is of some mathematical interest, but is of epidemiological interest only if it is false and 
there are differences in behavior for different choices of $\mathrm{P}(\mathrm{s})$. At this point, we must remark that a differential-difference model is not completely formulated until the initial conditions are given. The situation is different than for ordinary differential equations as the proper formulation of a delaydifferential equations requires the specification of initial conditions over the delayed period, that is, we must specify a real valued function that is defined over $[-\tau, 0]$. From the theory of differentialdifference equations it is well known that solutions will exist under very general conditions for almost all initial conditions. The situation may be different for integral equations, which are, in fact, more general than differential-difference equations. The problem of existence of solutions is more delicate, and it is intimately connected to the kernel $\mathrm{P}(\mathrm{s})$. Sometimes it is not possible to prescribe initial conditions, and sometimes solutions may not exist for almost all initial conditions. So when we claim that in some sense the above two choices represent two extremes, we are in fact providing a practical rule of thumb rather than a precise mathematical statement. However, this rule works quite well in some situations. The HIV/AIDS models analyzed by Castillo-Chavez et al. (1989b, c, d) provide a good example.

It is also possible to introduce an exposed period of fixed length $\omega$, or more generally with a probability $Q(s)$ of remaining in the exposed class a time $s$ after becoming exposed. The analysis of disease models with exposed and infective periods of fixed length reduces to the analysis of systems of differential-difference equations with two delays, and this leads to questions of whether all roots of a transcendental characteristic equation have a negative real part. We shall describe an example namely, an $\mathrm{S}-\mathrm{E}-\mathrm{I}-\mathrm{R}$ model (where $\mathrm{E}$ denotes the exposed class, that is, individuals that have acquired the disease but are not yet infectious). The model without an exposed class is a special case with the exposed period $\omega$ equal to zero.

Example: The $\mathrm{S}-\mathrm{E}-\mathrm{I}-\mathrm{R}$ model, without births or deaths where $\mathrm{S}+\mathrm{E}+\mathrm{I}+\mathrm{R} \equiv \mathrm{K}$.

The $\mathrm{S}-\mathrm{E}-\mathrm{I}-\mathrm{R}$ model with an exposed period of fixed length $\omega$ and an infective period of fixed length $\tau$ is

$$
\begin{gathered}
\mathrm{S}^{\prime}(\mathrm{t})=-\beta \mathrm{S}(\mathrm{t}) \frac{\mathrm{I}(\mathrm{t})}{\mathrm{K}} \\
\mathrm{E}^{\prime}(\mathrm{t})=\beta \mathrm{S}(\mathrm{t}) \frac{\mathrm{I}(\mathrm{t})}{\mathrm{K}}-\beta \mathrm{S}(\mathrm{t}-\omega) \frac{\mathrm{I}(\mathrm{t}-\omega)}{\mathrm{K}}, \text { or } \mathrm{E}(\mathrm{t})=\int_{\mathrm{t}-\omega}^{\mathrm{t}} \beta \mathrm{S}(\mathrm{x}) \frac{\mathrm{I}(\mathrm{x})}{\mathrm{K}} \mathrm{d} x \\
\mathrm{I}^{\prime}(\mathrm{t})=\beta \mathrm{S}(\mathrm{t}-\omega) \frac{\mathrm{I}(\mathrm{t}-\omega)}{\mathrm{K}}-\beta \mathrm{S}(\mathrm{t}-\tau-\omega) \frac{\mathrm{I}(\mathrm{t}-\tau-\omega)}{\mathrm{K}}, \text { or } \mathrm{I}(\mathrm{t})=\int_{\mathrm{t}-\tau-\omega}^{\mathrm{t}-\omega} \beta \mathrm{S}(\mathrm{x}) \frac{\mathrm{I}(\mathrm{x})}{\mathrm{K}} \mathrm{d} x \\
\mathrm{R}^{\prime}(\mathrm{t})=\beta \mathrm{S}(\mathrm{t}-\tau-\omega) \frac{\mathrm{I}(\mathrm{t}-\tau-\omega)}{\mathrm{K}} .
\end{gathered}
$$


Since $E$ and $R$ are determined when $S$ and $I$ are known, this can be reduced to the pair of equations

$$
\begin{gathered}
S^{\prime}(t)=-\beta S(t) \frac{I(t)}{K}, \\
I(t)=\int_{t-\tau-\omega}^{t-\omega} \beta S(x) \frac{I(x)}{K} d x
\end{gathered}
$$

and then to a single equation by writing

$$
\mathrm{I}(\mathrm{t})=-\int_{\mathrm{t}-\tau-\omega}^{\mathrm{t}-\omega} \mathrm{S}^{\prime}(\mathrm{x}) \mathrm{d} \mathrm{x}=\mathrm{S}(\mathrm{t}-\tau-\omega)-\mathrm{S}(\mathrm{t}-\omega)
$$

and substituting into $S^{\prime}(t)=-\beta S(t) \frac{I(t)}{K}$ to obtain

$$
S^{\prime}(t)=-\beta S(t) \frac{[S(t-\tau-\omega)-S(t-\omega)]}{K} .
$$

To this single differential-difference equation with two delays we must add some initial data for $(\tau+\omega) \leq \mathrm{t} \leq 0$ to give a well-posed problem, because every constant is a solution of the differentialdifference equation. It is possible to prove that $\mathrm{S}(\mathrm{t})$ is non-increasing and tends to a limit $\mathrm{S}_{\infty}>0$ as $\mathrm{t} \rightarrow \infty$; the limiting value will depend on the initial data. The analysis of this model is considerably more difficult than the analysis of the original Kermack-MacKendrick S - I - R model, but the results are qualitatively the same, that is, there is a threshold number that determines whether or not there is an epidemic.

Obviously, diseases for which variable and long infectious periods are important must be modeled using the approach of this section. An example is provided by the modeling of the sexual transmission of the HIV/AIDS virus in a homosexually-active population. Castillo-Chavez et al. (1989b, c, d) have shown that the results are qualitatively the same for all incubation period distributions (also see Blythe and Anderson, 1988a, b). However, the results may be different if the transmission probability per (sexual) contact, that is $\mathrm{p}$, depends on how long an individual has been infected (see Thieme and Castillo-Chavez 1989, 1993). In some sense, this is the kind of results that one may expect from mathematical models, that is, their use helps us focus on factors that do affect the qualitative dynamics of a disease. For HIV/AIDS in a homosexually-active and homogeneously mixing population only the age of infection has the potential of changing the qualitative dynamics. Of course, epidemiologists are also interested in quantitative results (predictions). These results are more difficult to obtain as specific data is needed. Furthermore, the above discussion makes it clear that quantitative predictions under a simple epidemic model (which requires less parameters and hence less data) would be based on specific assumptions, such as homogeneous mixing (all individuals behave in the same average way), and the functional form of the contact function $\phi(\mathrm{N})$. However, the same situation is true if one uses 
statistical models. For long-term predictions dynamic models seem to be the best choice while for short-term predictions statistical models are sufficient. This division is somewhat simplistic as other methods are available such as time series analysis. Efforts to combine time series analysis, statistical methods, and dynamical systems have been the focus of intensive research in epidemiology (or more generally in theoretical biology). For some recent results on this active area of research, we recommend the articles by Ellner (1989), Hastings et al. (1993), and references therein.

\section{Models with social structure}

Classical mathematical models in demography (Leslie 1945; Lotka 1922; MacKendrick 1926) concentrate on the dynamics of birth and death processes of female populations. They ignore the mating/marriage structures and their effect on social dynamics. The incorporation of mating structures or marriage functions was pioneered by both Kendall (1949) and Keyfitz (1949). Their work was extended by Fredrickson (1971), McFarland (1972), Parlett (1972), and Pollard (1973) two decades ago but with very limited impact. The HIV/AIDS epidemic attracted theoreticians' attention to the study of the effects of social dynamics on the spread of epidemics. Questions raised primarily by researchers interested in HIV/AIDS epidemiology have brought back interest in the modeling of marriage functions and their connection to social dynamics (Anderson et al. 1989; Blythe and CastilloChavez 1989, 1990; Blythe et al. 1991, 1992; Busenberg and Castillo-Chavez 1989, 1991; CastilloChavez 1989; Castillo-Chavez et al. 1989d; Castillo-Chavez et al. 1991; Dietz 1988; Dietz and Hadeler 1988; Gupta et al. 1989; Hadeler 1989a, b; Hadeler and Nagoma 1990; Huang 1989; Huang et al. 1992; Hyman and Stanley 1988, 1989; Jacquez et al. 1988, 1989; May and Anderson 1989; Sattenspiel 1987a, b; Sattenspiel and Simon 1987; Sattenspiel and Castillo-Chavez 1990; Waldstätter 1989). Research on the social spread of disease has grown at a very fast pace over the last decade.

The contact or social structure of a population plays a fundamental role on the transmission dynamics of diseases, cultural traits, genetic traits, etc. In the past, it has been modeled by assuming that the rate of transmission of the trait in consideration is directly proportional to the product of the number of those that have the trait and those that do not, that is, it is assumed the the incidence rate is a bilinear function of susceptibles and infecteds (see Anderson 1982; Anderson and May 1991; Bailey 1975; and references therein). The assumption that the rate of new "cases" (the incidence) is proportional to the product of "susceptibles" and "converts" (those infected), the mass-action law, is useful but only as described before in limited circumstances. In fact, it is not useful in the modeling of sexually transmitted diseases (STD's). A thorough analysis of the modeling assumptions involved in the construction of the incidence rate or "force" of infection has been carried out in a systematic fashion by Busenberg and Castillo-Chavez $(1989,1991)$.

The importance of the contact process on frequency dependent systems was recognized by Ross as

early as 1911 in his work on malaria (see also Lotka, 1923). The contact/social structure of the 
population must respond to demographic/epidemiological changes in the population. A flexible framework for the modeling of population interactions is being developed because several questions of theoretical and practical importance cannot be properly studied under the existing framework. Some recent applications to this new framework include those to food web dynamics (Velasco-Hernandez and Castillo-Chavez 1993), and those to cultural dynamics (Lubkin and Castillo-Chavez 1993).

Busenberg and Castillo-Chavez $(1989,1991)$ defined the contact/social structures through mixing/pair-formation matrices. In addition, they provided a useful characterization of these matrices, which constitutes the basis of our further analysis. Following Castillo-Chavez and Busenberg (1991) we introduce this framework within a two-sex mixing population. We begin with some needed notation and definitions:

$$
\begin{aligned}
& \mathrm{p}_{i j}(\mathrm{t})=\text { probability that a male in group } \mathrm{i} \text { mixed with a female in group } \mathrm{j} \text { at time } \mathrm{t} \\
& \mathrm{q}_{j i}(\mathrm{t})=\text { probability that a female in group } \mathrm{j} \text { mixed with a female in group } \mathrm{i} \text { at time } \mathrm{t} \\
& \text { given that she mixed with somebody; } \\
& \mathrm{T}_{i}^{m}(\mathrm{t})=\text { number of males in group } \mathrm{i} \text { at time } \mathrm{t} \text {; } \\
& \mathrm{T}_{j}^{f}(\mathrm{t})=\text { number of females in group } \mathrm{j} \text { at time } \mathrm{t} \text {; } \\
& b_{i}^{m}=\text { average (assumed constant) number of female partners per group i male per time unit } \\
& \text { = per capita pair-formation rate for group i males; } \\
& \mathrm{b}_{j}^{f}=\text { average (assumed constant) number of male partners per group } \mathrm{j} \text { female per time unit } \\
& =\text { per capita pair-formation rate for group } \mathrm{j} \text { females. }
\end{aligned}
$$

Definition. $\left(\mathrm{p}_{i j}(\mathrm{t}), \mathrm{q}_{j i}(\mathrm{t})\right)$ is called a mixing/pair-formation matrix if and only if it satisfies the following properties at all times:

$$
\mathrm{p}_{i j}(\mathrm{t}) \geq 0 \text { and } \mathrm{q}_{j i}(\mathrm{t}) \geq 0 \text { for } i=1, \ldots, \mathrm{L}, j=1, \ldots, \mathrm{N}
$$

$$
\begin{aligned}
& \sum_{j=1}^{\mathrm{N}} \mathrm{p}_{i j}(\mathrm{t})=1 \text { for } i=1, \ldots, \mathrm{L} ; \sum_{i=1}^{\mathrm{L}} \mathrm{q}_{j i}(\mathrm{t})=1 \text { for } j=1, \ldots, \mathrm{N} . \\
& \mathrm{c}_{i} \mathrm{~T}_{i}^{m}(\mathrm{t}) \mathrm{p}_{i j}(\mathrm{t}) \frac{\sum_{j=1}^{\mathrm{N}} \mathrm{b}_{j} \mathrm{~T}_{j}^{f}(\mathrm{t})}{\sum_{j=1}^{\mathrm{N}} \mathrm{c}_{i} \mathrm{~T}_{i}^{m}(\mathrm{t})}=\mathrm{b}_{j} \mathrm{~T}_{j}^{f}(\mathrm{t}) \mathrm{q}_{j i}(\mathrm{t}) \text { for } i=1, \ldots, \mathrm{L}, j=1, \ldots, \mathrm{N} .
\end{aligned}
$$

(A4) $\mathrm{p}_{i j}(\mathrm{t}) \equiv \mathrm{q}_{j i}(\mathrm{t}) \equiv 0$ by definition if $\mathrm{c}_{i} \mathrm{~b}_{j} \mathrm{~T}_{i}^{m}(\mathrm{t}) \mathrm{T}_{j}^{f}(\mathrm{t})=0$ for some $i, \quad 1 \leq i \leq \mathrm{L}$, and/or some $j, 1 \leq j \leq \mathrm{N}$.

The fraction in Property (A3) is the total sexual-activity ratio between females and males. This ratio is obviously not equal to 1 at all times. In fact, it expresses the fact that the total average rate of pair formation between males of type $i$ and females of type $j$ must be equal. A possible interpretation of our explicit formulation of the equality of these rates is that we assume that the partnership system 
favors females (that is, they will tend to achieve their desire optimal average) while males will have to modify their desired optimal rates $\left(c_{i}\right.$ 's) to take into account the total sexual-activity ratio. Or in other words, if we assume that the average pairing rates for females are constant then the male pairing rates cannot be arbitrary. Property (A4) asserts that individuals from populations that do not interact cannot possibly mix. An immediate consequence of the above properties is that the total effective average rates of male and female activity must agree at all times, that is,

$$
\sum_{i=1}^{\mathrm{L}} \mathrm{C}_{i} \mathrm{~T}_{i}^{m}=\sum_{j=1}^{\mathrm{N}} \mathrm{b}_{j} \mathrm{~T}_{j}^{f}
$$

where

$$
\mathrm{C}_{i} \equiv \mathrm{c}_{i} \frac{\sum_{j=1}^{\mathrm{N}} \mathrm{b}_{j} \mathrm{~T}_{j}^{f}(\mathrm{t})}{\sum_{j=1}^{N} \mathrm{c}_{i} \mathrm{~T}_{i}^{m}(\mathrm{t})} .
$$

The only separable solution to (A1) - (A4) is the Ross solution (named after Ross in recognition of his implicit knowledge of the above axioms, see Lotka $(1922))$ given by $\left(\left(\bar{p}_{j}(t), \bar{q}_{i}(t)\right)\right.$, where

$$
\overline{\mathrm{p}}_{j}(\mathrm{t})=\frac{\mathrm{b}_{j}^{f} \mathrm{~T}_{j}^{f}(\mathrm{t})}{\sum_{i=1} \mathrm{~b}_{i}^{f} \mathrm{~T}_{i}^{f}(\mathrm{t})}, \quad \overline{\mathrm{q}}_{i}(\mathrm{t})=\frac{\mathrm{b}_{i}^{m} \mathrm{~T}_{i}^{m}(\mathrm{t})}{\sum_{k=1} \mathrm{~b}_{j}^{m} \mathrm{~T}_{j}^{m}(\mathrm{t})} .
$$

Castillo-Chavez and Busenberg (1991) characterized all solutions to axioms (A1)-(A4) as multiplicative perturbations of the Ross solution. These perturbations are defined in terms of two matrices, $\Phi^{m}=\left\{\phi_{i j}^{m}\right\}$ and $\Phi^{f}=\left\{\phi_{j i}^{f}\right\}$. The matrices $\Phi^{m}$ and $\Phi^{f}$ define the preferences and/or affinities of types of individuals of one gender for other types (here of the opposite gender), and these preferences may change with time directly or through changes in the frequency of the types. We refer to these two matrices as the male and female preference matrices, respectively. To formulate this representation theorem the following expressions are needed:

$$
\begin{array}{lll}
\ell_{i}^{m} \equiv \sum_{j=1}^{\mathrm{N}} \overline{\mathrm{p}}_{j} \phi_{i j}^{m}, & \mathrm{R}_{i}^{m} \equiv 1-\ell_{i}^{m}, & \mathrm{~V}^{m} \equiv \sum_{i=1}^{\mathrm{L}} \overline{\mathrm{q}}_{i} \mathrm{R}_{i}^{m}, \\
\ell_{j}^{f} \equiv \sum_{i=1}^{\mathrm{L}} \overline{\mathrm{q}}_{i} \phi_{j i}^{f}, & \mathrm{R}_{j}^{f} \equiv 1-\ell_{j}^{f}, & \mathrm{~V}^{f} \equiv \sum_{j=1}^{\mathrm{N}} \overline{\mathrm{p}}_{j} \mathrm{R}_{j}^{f} .
\end{array}
$$

\section{Theorem 1.}

For each marriage function $(\mathbf{P}, \mathbf{Q})$, matrices $\Phi^{m}$ and $\Phi^{f}$ can be found so that

$$
\mathrm{p}_{i j}=\overline{\mathrm{p}}_{j}\left[\frac{\mathrm{R}_{j}^{f} \mathrm{R}_{i}^{m}}{\mathrm{~V}^{f}}+\phi_{i j}^{m}\right] \quad \text { and } \quad \mathrm{q}_{j i}=\overline{\mathrm{q}}_{i}\left[\frac{\mathrm{R}_{i}^{m} \mathrm{R}_{j}^{f}}{\mathrm{~V}^{m}}+\phi_{j i}^{f}\right]
$$

with $0 \leq \mathrm{R}_{i}^{m} \leq 1$ and $0 \leq \mathrm{R}_{j}^{f} \leq 1$ for $i=1, \ldots, \mathrm{L}, \quad j=1, \ldots, \mathrm{N}, \quad$ and $\sum_{i=1}^{\mathrm{L}} \ell_{i}^{m} \overline{\mathrm{q}}_{i}<1$ and $\sum_{j=1}^{N} \ell_{j}^{f} \overline{\mathrm{p}}_{j}<1$ if and only if

$$
\phi_{i j}^{m}=\phi_{j i}^{f}+\mathrm{R}_{i}^{m} \mathrm{R}_{j}^{f}\left[\frac{1}{\mathrm{~V}^{m}}-\frac{1}{\mathrm{~V}^{f}}\right]
$$


Condition (4.3) shows the implicit frequency (and time) dependent relationship forced by (A3) between the elements of $\boldsymbol{\Phi}^{m}$ and $\boldsymbol{\Phi}^{f}$. Using vector notation,

$$
\overrightarrow{\overrightarrow{\mathbf{p}}}=\left(\begin{array}{c}
\overline{\mathrm{p}}_{1} \\
\vdots \\
\overline{\mathrm{p}}_{\mathrm{N}}
\end{array}\right) \quad \text { and } \quad \overrightarrow{\mathbf{q}}=\left(\begin{array}{c}
\overline{\mathrm{q}}_{1} \\
\vdots \\
\overline{\mathrm{q}}_{\mathrm{L}}
\end{array}\right)
$$

we can combine the constraints imposed by (4.3) through an implicit nonlinear relationship

$$
\boldsymbol{\Phi}^{m}=\psi\left(\overrightarrow{\overline{\mathbf{p}}}, \overrightarrow{\overline{\mathbf{q}}}, \boldsymbol{\Phi}^{f}, \boldsymbol{\Phi}^{m}\right)
$$

where the elements of $\psi$ are defined component-wise by (4.3).

We proceed to outline some useful results (see Hsu Schmitz et al. 1993; Hsu Schmitz 1994) which gives an insight into the role of $\Phi^{m}$ and $\Phi^{f}$ :

\section{Theorem 2.}

If either $\phi_{i j}^{m}=\alpha, \quad 0 \leq \alpha<1 \quad \forall i, j, \quad$ or $\phi_{j i}^{f}=\beta, \quad 0 \leq \beta<1 \quad \forall j$, i, where $\alpha$ and $\beta$ are constants, then $\mathrm{p}_{i j}=\overline{\mathrm{p}}_{j}$ and $\mathrm{q}_{j i}=\overline{\mathrm{q}}_{i}$. That is, Equation (4.2) reduces to the unique separable Ross solution in (2).

It may be argued that this representation just passes the buck by transferring the difficulties from one set of matrices, $(\mathbf{P}, \mathbf{Q})$, to another, $\left(\boldsymbol{\Phi}^{m}, \boldsymbol{\Phi}^{f}\right)$. However, the use of preference matrices $\left(\boldsymbol{\Phi}^{m}, \boldsymbol{\Phi}^{f}\right)$ helps increase our understanding of the marriage/social structure of a population because they facilitate the modeling of specific, non-trivial mixing patterns between subpopulations. Our data (see Rubin et al 1992; Castillo-Chavez et al. 1992; Hsu Schmitz and Castillo-Chavez 1993, 1994; Hsu Schmitz 1994) shows that females mix with older males and males mix with younger females. In the past, these mixing patterns were not modeled because either they led to intractable mathematical models or there was no obvious way of doing it. The use of affinity matrices $\left(\Phi^{m}, \Phi^{f}\right)$ facilitates the construction of these "unusual" mating/social structures. The following result (Hsu Schmitz et al. 1993; Hsu Schmitz 1994) provides a parametric family of mixing matrices that allows to model age-dependent mixing.

Theorem 3. $\mathrm{V}^{f}=\mathrm{V}^{m}$ if and only if $\boldsymbol{\Phi}^{m}=\left(\boldsymbol{\Phi}^{f}\right)^{\mathrm{T}}$, where $\mathrm{T}$ denotes transposition.

The above result implies that the only solutions to axioms (A1)-(A4) with frequency-independent 
$\Phi^{m}$ and $\Phi^{f}$ are those with $\Phi^{m}=\left(\Phi^{f}\right)^{\mathrm{T}}$. This situation occurs when males and females have matching preferences that do not change with the dynamics of $\mathrm{T}_{i}^{m}(\mathrm{t})$ and $\mathrm{T}_{j}^{f}(\mathrm{t})$. Although the class of solutions with $\Phi^{m}=\left(\Phi^{f}\right)^{\mathrm{T}}$ is quite restrictive, this class extends, considerably, the mixing/mating structures available in the literature. If we use constant preference matrices $\boldsymbol{\Phi}^{m}$ and $\boldsymbol{\Phi}^{f}$, then the class of parametric mixing models generated with this selection becomes quite rich and flexible. Figure 2 shows a real mixing matrix while Figure 3 shows the corresponding Ross solutions. Both were constructed using our data from a known population of undergraduate students and their partners (see Rubin et al 1991; Castillo-Chavez et al. 1992; Hsu Schmitz and Castillo-Chavez 1993, 1994; Hsu Schmitz 1994) and both can be fit to matrices that satisfy the relationship $\Phi^{m}=\left(\Phi^{f}\right)^{\mathrm{T}}$.

Theorem 2 requires the relationship (4.3). This relation implies the existence of a function $\psi$ such that

$$
\boldsymbol{\Phi}^{m}=\psi\left(\overrightarrow{\overline{\mathbf{p}}}, \overrightarrow{\mathbf{q}}, \boldsymbol{\Phi}^{f}, \boldsymbol{\Phi}^{m}\right)
$$

or, in other words, the preferences of males for females and viceversa satisfy a complex relationship. Common sense dictates that if the set of preference of one gender (e.g., $\left.\Phi^{f}\right)$ is known then so must be the other (e.g., $\boldsymbol{\Phi}^{m}$ ) and in fact it can be shown (see Hsu Schmitz and Castillo-Chavez 1993a; Hsu Schmitz 1994) that this is the case. Hence there exists a function $\Psi$ such that

$$
\boldsymbol{\Phi}^{m}=\Psi\left(\overrightarrow{\mathbf{p}}, \overrightarrow{\overline{\mathbf{q}}}, \boldsymbol{\Phi}^{f}\right)
$$

This result will be referred as the " $\mathrm{T}$ " Theorem" because it reminds us that, in all situations, it "Takes Two to Tango" (this theorem got this colorful name from Stavros Busenberg). In this Section we assumed that the pair-formation rates $b_{i}^{m}$ and $b_{j}^{f}$ are both constants. This was done to simplify the exposition because a consistent theory with constant pair-formation rates for both genders only holds under very particular circumstances. In general one has that $\mathrm{b}_{i}^{m} \equiv \mathrm{b}_{i}^{m}\left(\mathrm{~T}_{j}^{f}, \mathrm{~T}_{i}^{m}\right)$ and $\mathrm{b}_{j}^{f} \equiv \mathrm{b}_{j}^{f}\left(\mathrm{~T}_{j}^{f}\right.$, $\mathrm{T}_{i}^{m}$ ). For a further discussion of this general case the reader is referred to Castillo-Chavez et al. (1992, 1994b), Hsu Schmitz and Castillo-Chavez (1993, 1994) and Hsu Schmitz (1994). Castillo-Chavez et al. (1994b) shows explicit ways of incorporating arbitrary mixing patterns into demographic and epidemiological models. The mathematical analysis of these general models is progressing (see Huang 
1989; Huang et al. 1993). Section 5 deals with applications of this framework in an stochastic setting. We formulate a stochastic pair-formation model for a treatable sexually-transmitted diseases based on the deterministic epidemic model presented in Blythe et al. (1991).

\section{Stochastic Models with social structure}

In this section, we formulate a stochastic pair-formation epidemiological model by using the approach that is common to interacting particle systems (for details, see Luo and Castillo-Chavez 1991; Castillo-Chavez et al. 1994a, 1994b). Extensions of this model to cover other scenarios should be quite evident from the following description.

We define

$$
\mathrm{X}=\{0,1, \cdots, \mathrm{L}\} \times\{0,1\} \times\{0,1, \cdots, \mathrm{N}\} \times\{0,1\} /\{0\} \times\{0,1\} \times\{0\} \times\{0,1\},
$$

and consider the explicit stochastic process

$$
\xi_{t}: \mathrm{X} \rightarrow\{0,1,2, \cdots\}, \quad \mathrm{t} \geq 0 .
$$

Let $x=(i, u ; j, v) \in X$, where $i$ and $j$ denote the groups of males and females and $u$ and $v$ denote the epidemiological statuses of males and females, respectively. If we consider an STD that does not have a long latent period, does not provide permanent immunity, and does not cause significant mortality (e.g., gonorrhea, see Hethcote and Yorke 1984), then the possible values of $u$ and $v$ are either 0 (susceptible) or 1 (infected). For $\mathrm{i}>0$ and $\mathrm{j}>0, \mathrm{x}$ gives the type of pair; that is, the male is from group $i$ with epidemiological status $u$ and the female is from group $j$ with epidemiological status $v$. If $i=0$ but $j>0$, then $x$ represents a single female in group $j$ with epidemiological status $v$ (the value of $u$ is not relevant) and therefore we can define $x \equiv(0 ; j, v) \equiv(0,0 ; j, v) \equiv(0,1 ; j, v)$. Similarly, if $j=0$ but $\mathrm{i}>0$, then $\mathrm{x}$ represents a single male in group $\mathrm{i}$ with epidemiological status $\mathrm{u}$ and again we can define $\mathrm{x} \equiv(\mathrm{i}, \mathrm{u} ; 0) \equiv(\mathrm{i}, \mathrm{u} ; 0,0) \equiv(\mathrm{i}, \mathrm{u} ; 0,1)$. Note that the case of $\mathrm{i}=\mathbf{0}$ and $\mathrm{j}=\mathbf{0}$ is not included in the domain of $\mathrm{X}$. Consequently, the stochastic process $\xi_{t}(\mathrm{x})$ gives the number of pairs of type $\mathrm{x}$ at time $\mathrm{t}$ if $i>0$ and $j>0$; it gives the number of single males of type $x$ at time $t$ if $i>0$ and $j=0$; and it gives the number of single females of type $x$ at time $t$ if $i=0$ and $j>0$.

To complete the characterization of $\xi_{t}(\mathrm{x})$, we define $\mathrm{S} \equiv\{0,1,2, \ldots\}^{X}$ and let $\mathrm{c}: \mathrm{S} \times \mathrm{S} \rightarrow(0, \infty)$ be a real-valued function that models the flip rate. We view $\left\{\xi_{t}: t \geq 0\right\}$ as an S-valued Markov process 
with flip rate $c(\cdot, \cdot)$, i.e., if $\xi_{t}=\xi$ for some $\mathrm{t} \geq 0$, then $\mathrm{c}(\xi, \eta)$ denotes the instantaneous rate at which $\xi_{t}$ may change to state $\eta$. Explicitly,

$$
\operatorname{Prob}\left(\xi_{t+h}=\eta \mid \xi_{t}=\xi\right)=\mathrm{c}(\xi, \eta) \mathrm{h}+\mathrm{o}(\mathrm{h}), \quad \forall \mathrm{t} \geq 0 .
$$

The more specific definition of flip rates is as follows: for $\xi \in S, A \subset X, B \subset X$, and $A \cap B=\emptyset$, we define $\xi_{B}^{A}(\mathrm{x}) \in \mathrm{S}$ as

$$
\xi_{B}^{A}(\mathrm{x})= \begin{cases}\xi(\mathrm{x})+1 & \text { if } \mathrm{x} \in \mathrm{A} \\ \xi(\mathrm{x})-1 & \text { if } \mathrm{x} \in \mathrm{B} \\ \xi(\mathrm{x}) & \text { otherwise }\end{cases}
$$

Thus, the system $\left\{\xi_{t}\right\}$ consists of a series of changing elements in the set $\mathrm{S}$, which is the set of all functions on $\mathrm{X}$. The dynamics of the system is described by the rates $\{c(\xi, \eta): \xi \neq \eta, \xi, \eta \in S\}$ at which the system changes.

We assume the existence of an underlying mixing/pair-formation matrix $\left(\mathrm{p}_{i j}\left(\xi_{t}\right), \mathrm{q}_{j i}\left(\xi_{t}\right)\right)$ as described in Section 4. Since $\xi_{t}$ is a function of $t$, the mixing matrix is also a function of $t$. We further assume that paired individuals do not look for other partners before they separate. As the time $t$ changes, singles may form pairs, pairs may dissolve, the disease may be transmitted within pairs from an infective to a susceptible, the infectives may be cured, etc.

We use the indices $m$ and $f$ to identify the parameters associated with males and females regardless of their epidemiological status, and use $\mathrm{M}$ and $\mathrm{F}$ to characterize those parameters only associated with infected males and females, respectively. Then the flip rate $c(\cdot, \cdot)$ is calculated as follows:

a) Pair formation

For $\mathrm{i}>0, \mathrm{j}>0$,

$$
\begin{aligned}
\mathrm{c}\left(\xi, \xi \xi_{i, u ; 0),(0 ; j, v)}^{i, u ; j, v)}\right. & =\mathrm{b}_{j}^{f} \xi(0 ; \mathrm{j}, \mathrm{v}) \mathrm{q}_{j i}(\xi) \frac{\xi(\mathrm{i}, \mathrm{u} ; 0)}{\xi(\mathrm{i}, \mathrm{u} ; 0)+\xi(\mathrm{i}, 1-\mathrm{u} ; 0)} \\
& =\mathrm{b}_{i}^{m} \xi(\mathrm{i}, \mathrm{u} ; 0) \mathrm{p}_{i j}(\xi) \frac{\xi(0 ; \mathrm{j}, \mathrm{v})}{\xi(0 ; \mathrm{j}, \mathrm{v})+\xi(0 ; \mathrm{j}, 1-\mathrm{v})}
\end{aligned}
$$

b) Pair dissolution ( $\sigma$ denotes the constant pair dissolution rate)

For $\mathrm{i}>0, \mathrm{j}>0$,

$$
\mathrm{c}\left(\xi, \xi\left(\begin{array}{l}
i, u ; j, j, v) \\
(i, j, j, v)
\end{array}\right)=\sigma_{i j} \xi(\mathrm{i}, \mathrm{u} ; \mathrm{j}, \mathrm{v})\right.
$$

c) Transmission ( $\delta$ denotes the constant transmission rate) 
For $\mathrm{i}>0, \mathrm{j}>0$,

$$
\mathrm{c}\left(\xi, \xi\left(\begin{array}{l}
i, 1 ; j, 1) \\
i, 0 ; j, 1)
\end{array}\right)=\delta_{F} \xi(\mathrm{i}, 0 ; \mathrm{j}, 1), \quad \mathrm{c}\left(\xi, \xi\left(\begin{array}{l}
i, 1 ; j ; j, 1) \\
(i, 1 ; j)
\end{array}\right)=\delta_{M} \xi(\mathrm{i}, 1 ; \mathrm{j}, 0)\right.\right.
$$

d) Recovery ( $\gamma$ denotes the constant recovery rate)

For $\mathrm{i}>0, \mathrm{j}>0$, the recovery flip rates for one paired individual are

$$
\begin{aligned}
& \mathrm{c}\left(\xi, \xi_{(i, 0 ; j, 1)}^{(i, 0 ; j, 0)}\right)=\gamma_{F} \xi(\mathrm{i}, 0 ; \mathrm{j}, 1), \quad \mathrm{c}\left(\xi, \xi_{(i, 1 ; j, 0)}^{(i, 0 ; j, 0)}\right)=\gamma_{M} \xi(\mathrm{i}, 1 ; \mathrm{j}, 0) ; \\
& \mathrm{c}\left(\xi, \xi_{(i, 1 ; j, 1}^{(i, 1 ; j, 0)}\right)=\gamma_{F} \xi(\mathrm{i}, 1 ; \mathrm{j}, 1), \quad \mathrm{c}\left(\xi, \xi_{(i, 1 ; j, 1}^{(i, 0 ; j, 1)}\right)=\gamma_{M} \xi(\mathrm{i}, 1 ; \mathrm{j}, 1) ;
\end{aligned}
$$

and the flip rate for both individuals is

$$
\mathrm{c}\left(\xi, \xi\left(\begin{array}{l}
i, 0 ; j ; j, 1) \\
(i, j)
\end{array}\right)=\gamma_{F M} \xi(\mathrm{i}, 1 ; \mathrm{j}, 1) ;\right.
$$

while for single infected individuals $(j=0$ or $i=0)$ we have

$$
\mathrm{c}\left(\xi, \xi_{(i, 1 ; 0)}^{(i, 0 ; 0)}\right)=\gamma_{M} \xi(\mathrm{i}, 1 ; 0), \quad \mathrm{c}\left(\xi, \xi_{(0 ; j, 1)}^{(0 ; j, 0)}\right)=\gamma_{F} \xi(0 ; \mathrm{j}, 1)
$$

e) Removal ( $\mu$ denotes the constant removal rate from sexual activity)

For $\mathrm{i}>0, \mathrm{j}>\mathbf{0}$,

$$
\mathrm{c}\left(\xi, \xi_{(i, u ; j, v)}^{(i, u ; 0)}\right)=\mu_{f} \xi(\mathrm{i}, \mathrm{u} ; \mathrm{j}, \mathrm{v}), \quad \mathrm{c}\left(\xi, \xi_{(i, u ; j, v)}^{(0 ; j, v)}\right)=\mu_{m} \xi(\mathrm{i}, \mathrm{u} ; \mathrm{j}, \mathrm{v})
$$

while for single individuals $(j=0$ or $i=0)$ we have

$$
\mathrm{c}\left(\xi, \xi_{(i, u ; 0)}\right)=\mu_{m} \xi(\mathrm{i}, \mathrm{u} ; 0), \quad \mathrm{c}\left(\xi, \xi_{(0 ; j, v)}\right)=\mu_{f} \xi(0 ; \mathrm{j}, \mathrm{v})
$$

f) Recruitment ( $\Lambda$ denotes the constant recruitment rate for susceptible singles)

For $\mathrm{i}>0, \mathrm{j}>0$,

$$
\mathrm{c}\left(\xi, \xi^{(i, 0 ; 0)}\right)=\Lambda_{i}^{m} \quad \text { and } \quad c\left(\xi, \xi^{(0 ; j, 0)}\right)=\Lambda_{j}^{f}
$$

g) Other

For any other $\eta \neq \xi$, we assume $c(\xi, \eta)=0$.

h) $\mathrm{c}(\xi, \xi)=\sum_{\eta \neq \xi} \mathrm{c}(\xi, \eta)$.

This concludes the characterization of our stochastic epidemiological model with pairs. Next, we outline the simulation procedure of a general stochastic process including the above. From the construction of the flip rates we know that

$$
c(\xi)=\sum_{\eta \neq \xi} c(\xi, \eta)<\infty .
$$

If we let the sequence $0=\rho_{0}<\rho_{1}<\cdots$ denote the jump times of the process, then $\tau_{n}=\rho_{n}-\rho_{n-1}$ has an exponential distribution with rate $\mathrm{c}\left(\xi_{\rho_{n-1}}\right)$. Thus, the process can be simulated as follows: 
1) First, set the initial state $\xi_{0}$ and assume that a sequence of n jump times $0=\rho_{0}<\rho_{1}<\cdots<\rho_{n}$ and their corresponding states $\xi_{\rho_{i}}, 1 \leq \mathrm{i} \leq \mathrm{n}$, have been determined.

2) Get $\tau_{n+1}$ from $\exp \left\{\xi_{\rho_{n}}\right\}$ and let $\rho_{n+1} \equiv \rho_{n}+\tau_{n+1}$.

3) Set $\xi_{\rho_{n+1}}=\eta$ with probability $\mathrm{c}\left(\xi_{\rho_{n}}, \eta\right) / \mathrm{c}\left(\xi_{\rho_{n}}\right)$.

4) Define $\xi_{t}=\xi_{\rho_{n}}$ for $\rho_{n} \leq \mathrm{t}<\rho_{n+1}$.

In Castillo-Chavez et al. (1994a) extensive simulations are carried out for pair-formation demographic models where individuals have distinct preferences. We found (later justified analytically in some simple cases in Hsu Schmitz, 1994) that the parameters with a bigger impact on the determination of the stable pair distribution are the dissolution rates. If these rates are identical for all groups then the system stabilizes asymptotically to a pair-distribution that is in agreement with individual preferences. However, if the dissolution rates differ, then there is no clear connection between the "observed" mating preferences/affinities and the observed mixing patterns (stable pairdistribution). This is quite analogous to what is observed in population genetics where there is no unique correspondence between genotype and phenotype. In fact, higher variance on the dissolution rates may reduce our ability to identify individual affinities from observed mixing patterns.

\section{Conclusions.}

In this chapter we provide an overview on the use, formulation, and analysis of epidemiological models. The introduction briefly touches on ways in which models can help us understand the mechanisms behind the (underlying) dynamics of observed time series (see Hethcote and Levin 1989; Castillo-Chavez et al. 1988, 1989 for specific examples). Section 2 provides a concise introduction to a large class of simple and useful epidemiological models. A complete mathematical analysis of these models is not provided but, we take the time to illustrate the role that the basic reproductive number plays in epidemiology.

The HIV/AIDS epidemic has forced us to look at models that incorporate long and variable periods of infectiousness and hence, we must deal with systems of difference-differential and integral equations (the topic of Section 3). These models are more difficult to handle but their analysis has been carried out in many situations. In fact, there has been a great deal of progress since the first articles on the transmission dynamics of HIV (see Anderson et al. 1986; Anderson and May 1987; Pickering et al. 1986) appeared almost a decade ago.

If one wishes to construct more realistic models for the transmission dynamics of HIV/AIDS then one has to incorporate other factors including the social structure of a population. Section 4 outlines 
ways in which this can be accomplished as well as some of the difficulties encountered in the process. To limit the level of complexity, we abandon epidemics and concentrate on the development of methods for modeling the (dynamic) contact/pairing structure of a population. The usefulness of our approach has yet to be determined albeit the fact, that it has already been applied with some degree of success to data on mixing patterns of college undergraduates (see Blythe et al. 1992; Castillo-Chavez et al. 1992; Rubin et al. 1992; Hsu Schmitz and Castillo-Chavez 1994, Hsu Schmitz et al. 1993, Hsu Schmitz 1994). Section 5 deals with the formulation of stochastic pair-formation epidemic models. The analysis of stochastic models is difficult but their simulation can be easily carried out (we provide an outline of the simulation method). References to the extensive work that is being carried out on (the very active field of) stochastic epidemics can be found in Gabriel et al. (1990), Castillo-Chavez et al. (1994a,b), and Luo and Castillo-Chavez (1991).

This chapter focussed on modeling rather than on mathematics yet we have not completely run away from all the technicalities. In fact, we have tackled some of them while providing extensive references to those wishing to learn the basic tricks. However, the time used on this chapter will not be well spent unless it helps the reader formulate (preferably simple) models that are capable of addressing important biological questions. The references provide ample documentation of the successes that mathematics has had when it is driven by biological research.

\section{Acknowledgements.}

This research was partially supported by NSF grant DEB-925370 (Presidential Faculty Fellowship Award) to Carlos Castillo-Chavez and by the U.S. Army Research Office through the Mathematical Sciences Institute of Cornell University (contract DAAL03-91-C-0027). We thank Sam Fridman for his help with the Figures, Kety Esquivel for many thankless tasks, and John Steele for his inifinte patience. This manuscript was completed while Carlos Castillo-Chavez was a Fellow at the Department of Ecology and Evolutionary Biolgy in Princeton University. 


\section{References}

Allen J. C. (1989). Are natural enemy populations chaotic? In Estimation and Analysis of Insect Populations. Lecture Notes ins Statistics 55:190-205.

Allen J. C. (1989). Patch efficient parasitoids, chaos, and natural selection. Fla. Entomol. 79:52-64.

Allen J. C. (1990). Factors contributing to chaos in population feedback systems. Ecol. Model. 51:281-298.

Allen J. C. (1990). Chaos in phase-locking in predator-prey models in relation to the functional response. Fla. Entomo. 73:100-110.

Allen J. C. (1991). Chaos and coevolutionary warfare in a chaotic predator-prey system. Fla. Entomol. 74:50-59.

Altenberg L. (1991). Chaos from linear frequency-dependent selection. Am. Nat. 138:51-68.

Anderson, R.M., ed. (1982). Population Dynamics of Infectious Diseases: Theory and Applications. Chapman and Hall: London, New York.

Anderson, R. M. and R. M. May. (1987). Transmission dynamics of HIV infection, Nature, 326:137142.

Anderson, R.M., Blythe, S.P., Gupta, S. and Konings, E. (1989) The transmission dynamics of the Human Immunodeficiency Virus Type 1 in the male homosexual community in the United Kingdom: the influence of changes in sexual behavior. Phil. Trans. R. Soc. Lond. B 325, 145-198.

Anderson, R.M. and R.M. May. (1991). Infectious Diseases of Humans. Oxford Science Publications: Great Britain.

Anderson, R. M., R. M. May, G. F. Medley, and A. Johnson. (1986). A preliminary study of the transmission dynamics of the human immunodeficiency virus (HIV), the causative agent of AIDS, IMA. J. Math. Med. Biol. 229-263.

Bailey, N.T.J. (1975). The Mathematical Theory of Infectious Diseases and Its Applications. Griffin: London.

Beddington J. R., Free C. A., and Lawton J. H. (1975). Dynamic complexity in predator-prey models framed in difference equations. Nature 255:58-60.

Bellows T. S., Hassell M. P. (1988). The dynamics of age-structured host-parasitoid interactions. J. Anim. Ecol. 57:259-268.

Blythe, S. P. and R. M. Anderson. (1988a). Distributed incubation and infectious periods in models of the transmission dynamics of the human immunodeficiency virus (HIV). IMA J. Math. Med. Biol. 5:1-19. 
Blythe, S.P. and R.M. Anderson. (1988b). Variable infectiousness in HIV transmission models. IMA J. of Mathematics Applied in Med. and Biol. 5, 181-200.

Blythe, S.P. and C. Castillo-Chavez. (1989). Like-with-like preference and sexual mixing models. Math. Biosci. 96, 221-238.

Blythe, S. P. and Castillo-Chavez, C. (1990). Scaling law of sexual activity. Nature, 344: 202.

Blythe, S.P., C. Castillo-Chavez, J. Palmer, and M. Cheng. (1991). Towards a unified theory of mixing and pair formation. Math. Biosci. 107, 379-405.

Blythe, S. P., Castillo-Chavez, C., and Casella, G. (1992) Empirical methods for the estimation of the mixing probabilities for socially-structured populations from a single survey sample. Mathematical Population Studies. 3(3): 199-225.

Brauer, F. (1990). Models for the spread of universally fatal diseases. J. M. Biolo. 28: 451-462.

Brauer, F. (1991). Models for the spread of universally fatal diseases II. In: Proceedings of the International Conference on Differential Equations and Applications to Biology and Population Dynamics, S. Busenberg and M. Martelli (eds.), Lecture Notes in Biomathematics 92, 57-69. Berlin, Heidelberg, New York, London, Paris, Tokyo, Hong Kong, Barcelona, Budapest: Springer-Verlag.

Busenberg, S. and C. Castillo-Chavez. (1989). Interaction, pair formation and force of infection terms in sexually transmitted diseases. In (C. Castillo-Chavez, ed.) Mathematical and Statistical Approaches to AIDS Epidemiology. Lecture Notes in Biomathematics 83, Springer-Verlag, Berlin, Heidelberg, New York, London, Paris, Tokyo, Hong Kong: 289-300.

Busenberg, S. and C. Castillo-Chavez. (1991). A general solution of the problem of mixing subpopulations, and its application to risk- and age-structured epidemic models for the spread of AIDS. IMA J. of Math. Appl. Med. Biol. 8, 1-29.

Busenberg, S. and K. Cooke. (1993). Vertically transmitted diseases: Models and Dynamics, Biomathematics 23, Springer-Verlag, Berlin, Heidelberg, New York.

Capasso, V. (1993). Mathematical Structures of Epidemic Systems. Lecture Notes in Biomathematics 97, Springer-Verlag, Berlin, Heidelberg, New York.

Castillo-Chavez, C., ed. (1989). Mathematical and Statistical Approaches to AIDS Epidemiology Lecture Notes in Biomathematics, 83, Springer-Verlag: New York.

Castillo-Chavez, C., Hethcote, H., Andreasen, V., Levin, S. A., and Liu, W-m. (1988). Cross-immunity in the dynamics of homogeneous and heterogeneous populations. In: Mathematical Ecology (T. G. Hallam, L. G. Gross, and S. A. Levin, eds.). pp. 303-316. World Scientific Publishing Co., Singapore.

Castillo-Chavez, C., Hethcote, H., Andreasen, V., Levin, S. A., and Liu, W-m. (1989a). Epidemiological models with age structure, proportionate mixing, and cross-immunity. J. Math. Biol., 27(3): 233-258. 
Castillo-Chavez, C., Cooke, K., Huang, W. and Levin, S. A. (1989b). The role of long incubation periods in the dynamics of HIV/AIDS. Part 1: Single population models. J. Math. Biology, 27: 373-398.

Castillo-Chavez, C., Cooke, K., Huang, W. and Levin, S. A. (1989c). On the role of long incubation periods in the dynamics of HIV/AIDS. Part 2: Multiple group models. In: Mathematical and statistical approaches to AIDS epidemiology (C. Castillo-Chavez, ed.), pp. 200-217. Lecture Notes in Biomathematics 83, Springer-Verlag, Berlin, Heidelberg, New York, London, Paris, Tokyo, Hong Kong.

Castillo-Chavez, C., Cooke, K., Huang, W. and Levin, S. A. (1989d). Results on the dynamics for models for the sexual transmission of the human immunodeficiency virus. Applied Math. Letters, 2(4): 327-331.

Castillo-Chavez, C. and Busenberg, S. (1991). On the solution of the two-sex mixing problem. In: Proceedings of the International Conference on Differential Equations and Applications to Biology and Population Dynamics, S. Busenberg and M. Martelli (eds.), Lecture Notes in Biomathematics 92, 80-98. Berlin, Heidelberg, New York, London, Paris, Tokyo, Hong Kong, Barcelona, Budapest: Springer-Verlag.

Castillo-Chavez, C., Busenberg, S., and Gerow K. (1991). Pair formation in structured populations. In: Differential Equations with Applications in Biology, Physics and Engineering (J. Goldstein, F. Kappel, W. Schappacher, eds.), pp. 47-65. Marcel Dekker, New York.

Castillo-Chavez, C., Shyu, S-F., Rubin, G. and Umbauch, D. (1992) On the estimation problem of mixing/pair formation matrices with applications to models for sexually-transmitted diseases. In: AIDS Epidemiology: Methodology Issues (K. Dietz, V. T. Farewell, N. P. Jewell, eds.), 384402. Birkhäuser, Boston-Basel-Berlin.

Castillo-Chavez, C., S. Fridman, and X. Luo. (1994a). Stochastic and deterministic models in epidemiology. In: Proc. First World Congress on Nonlinear Analysts, Tampa, Florida, August 19-26, 1992. (In press).

Castillo-Chavez, C., J.X. Velasco-Hernandez, and S. Fridman. (1994b). Modeling Contact Structures in Biology. In: Frontiers of Theoretical Biology, S. A. Levin (ed.), Lecture Notes in Biomathematics, 100. Springer-Verlag: New York (in press).

Caswell H. and Weeks D. E. (1986). Two-sex models: Chaos, extinction, and other dynamic consequences of sex. Am. Nat. 128:707-735.

Cressman R. (1988). Complex dynamical behaviour of frequency dependent viability selection: an example. J. Theor. Bio. 130:167-173.

Diekmann, O., J.A.P. Heesterbeek, and J.A.J. Metz. (1990). On the definition of $\mathrm{R}_{0}$ in models for infectious diseases in heterogeneous populations. J. Mathematical Biology, 28:365-382.

Dietz, K. (1988). On the transmission dynamics of HIV. Math. Biosci. 90, 397-414. 
Dietz, K. and K.P. Hadeler. (1988). Epidemiological models for sexually transmitted diseases. J. Math. Biol. 26, 1-25.

Ebenman B. (1987). Niche differences between age classes and intraspecific competition in age structured populations. J. Theor. Biol. 124:25-33.

Ellner S. (1989). Inferring the causes of population fluctuations. In: Mathematical Approaches to Problems in Resource Management and Epidemiology (C. Castillo-Chavez, S. A. Levin, and C. A. Shoemaker eds.). Lecture Notes in Biomathematics 81, Springer-Verlag, Berlin, Heidelberg, and New York.

Evans A. S. (1982). Viral infections of humans (2nd ed.) Plenum Medical Book Company, New York.

Fredrickson, A.G. (1971). A mathematical theory of age structure in sexual populations: Random mating and monogamous marriage models. Math. Biosci. 20, 117-143.

Gabriel, J. P., Lefèvre, C., and Picard, P. (eds.) (1990). Stochastic Processes in Epidemic Theory. Lecture Notes in Biomathematics 86, Springer-Verlag, Berlin, Heidelberg, New York, London, Paris, Tokyo, Hong Kong.

Gardini L., Lupini R., Mammana C., and Messia M. G. (1987). Bifurcation and transition to chaos in the three dimensional Lotka Volterra map. SIAM. J. Appl. Math. 47:455-482.

Gupta S, Anderson RM, May RM. Network of sexual contacts: implications for the pattern of spread of HIV. AIDS 1989; 3: 1-11.

Gilpin M. E. (1992). Spiral chaos in a predator prey model. Am. Nat. 113:306-308.

Guckenheimer J., Oster G. and Ipaktchi A. (1977). The dynamics of density-dependent populations models. J. Math. Biol. 4:101-147.

Hadeler, K.P. (1989a). Pair formation in age-structured populations. Acta Applicandae Mathematicae 14, 91-102.

Hadeler, K.P. (1989b). Modeling AIDS in structured populations. In: Proc. 47th Session of the International Statistical Institute, Paris, August/September, pp. 83-99.

Hadeler, K.P. and K. Nagoma. (1990). Homogeneous models for sexually transmitted diseases. Rocky Mountain J. of Math. 20, 967-986.

Hastings A., Hom C. L., Ellner, S., Turchin P., and Godfray H. C. J. (1993). Chaos in Ecology: Is Mother Nature a Strange Attractor? Annu. Rev. Ecol. Syst. 24:1-33.

Hastings A. and Powell T. (1991). Chaos in a three species food chain. Ecology 72:896-903.

Hethcote, H.W. (1976). Qualitative analyses for communicable disease models, Math. Biosci. 28, 335356.

Hethcote, H.W. (1978). An immunization model for a heterogeneous population. Theor. Pop. Biol. $14,338-349$. 
Hethcote, H. W. (1989). Three Basic Epidemiological Models. In: Applied Mathematical Ecology, S. A. Levin, T. G. Hallam, and L. J. Gross (eds.), Biomathematics 18, 119-144, Springer-Verlag, Berlin, Heidelber, New York.

Hethcote, H.W. and S.A. Levin. (1989). Periodicity in epidemiological models. In: Applied Mathematical Ecology, S.A. Levin, T.G. Hallam, and L.J. Gross (eds.). Biomathematics 18, Springer-Verlag, Heidelberg.

Hethcote, H. W. and J. W. Van Ark (1992). Modeling HIV Transmission and AIDS in the United States. Lecture Notes in Biomathematics, Vol. 95. Springer-Verlag, New York.

Hethcote, H.W. and J.A. Yorke. (1984). Gonorrhea Transmission Dynamics and Control. Lecture Notes in Biomathematics 56. Springer-Verlag: New York.

Hochberg M. E., Hassell M. P., and May R. M. (1990). The dynamics of host-parasitoid-pathogen interactions. Am. Nat. 135:74-94.

Hsu Schmitz, S. F. (1994). Some theories, estimation methods, and applications of marriage and mixing functions to demography and epidemiology. Ph.D. dissertation, Cornell University, Ithaca, New York.

Hsu-Schmitz, S.-F. and C. Castillo-Chavez. (1993). Completion of mixing matrices for non-closed social networks. In Proc. First World Congress on Nonlinear Analysts, Tampa, Florida, August 19-26, 1992. (In press).

Hsu Schmitz, S-F. and Castillo-Chavez, C. (1994). Parameter estimation in non-closed social networks related to the dynamics of sexually-transmitted diseases. In: Modeling the AIDS epidemic. (Kaplan, E. and Brandeau, M. eds., Raven Press, New York (in press).

Hsu Schmitz, S-F., Busenberg, S., and Castillo-Chavez, C. (1993). On the evolution of marriage functions: It Takes Two to Tango. Biometrics Unit Technical Report BU-1210-M, Cornell University, Ithaca, New York.

Huang, W. (1989). Studies in differential equations and applications. Ph. D. Thessis, The Claremont Graduate School (December 1989), Claremont CA.

Huang, W., Cooke, K., and Castillo-Chavez, C. (1992). Stability and bifurcation for a multiple group model for the dynamics of HIV/AIDS transmission. SIAM J. of Applied Math., Vol 52, No. $3: 835-854$

Hyman, J.M. and E.A. Stanley. (1988). Using mathematical models to understand the AIDS epidemic. Math. Biosci. 90, 415-473.

Hyman, J.M. and E.A. Stanley. (1989). The effect of social mixing patterns on the spread of AIDS. In: Mathematical approaches to problems in resource management and epidemiology. C. CastilloChavez, S.A. Levin, and C. Shoemaker (eds.). Lecture Notes in Biomathematics 81, SpringerVerlag, Berlin, Heidelberg, New York, Tokyo: 190-219

Jacquez, J.A., C.P. Simon, J. Koopman, L. Sattenspiel, and T. Perry. (1988). Modelling and 
analyzing HIV transmission: the effect of contact patterns. Math. Biosci. 92, 119-199.

Jacquez, J.A., Simon, C.P. and Koopman, J. (1989). Structured mixing: heterogeneous mixing by the definition of mixing groups. Mathematical and Statistical Approaches to AIDS Epidemiology (C. Castillo-Chavez, ed.) Lecture Notes in Biomathematics 83, 301-315. Springer-Verlag.

Jewell, N.P., K. Dietz, and V.T. Farewell. (1991). AIDS Epidemiology: Methodological Issues. Birkhäuser: Boston, Basel, Berlin.

Kaplan. Ed. and M. Brandeau (eds.) (1994). Modeling AIDS the AIDS epidemic. Raven Press, New York (in press).

Kendall, D.G. (1949). Stochastic processes and population growth. Roy. Statist. Soc. Ser. B 2, 230264.

Kermack, W. O. and A. G. McKendrick. (1927). A contribution to the mathematical theory of epidemics. Proceedings Roy. Soc. London, Ser. A 115:700-721.

Kermack, W. O. and A. G. McKendrick. (1932). A contribution to the mathematical theory of epidemics. Proceedings Roy. Soc. London, Ser. A 138:55-83.

Keyfitz, N. (1949). The mathematics of sex and marriage. In: Proc. Sixth Berkeley Symp. Math. Statist. Prob. 4, 89-108.

Kot M., Schaffer W. M., Truty G. L., Graser D. J., and Olsen L. F. (1988). Changing criteria for imposing order. Ecol. Model. 43:75-110.

Leslie, P.H. (1945). On the use of matrices in certain population mathematics. Biometrika 33, 183212.

Levin S.A. (1981). Age structure and stability in multiple-age populations. Renewable Resource Management. Lecture Notes in Biomath. 40:21-45. (eds.)

Levin S. A. (1983a). Coevolution. In: Population Biology. Freedman, H. I. and Strobeck, C. (eds.) Lecture Notes in Biomathematics 52:328-324, Springer-Verlag, Heidelberg.

Levin S. A. (1983b). Some approaches in the modeling of coevolutionary interactions. In: Coevolution. M. Nitecki (ed.), 21-65, University of Chicago Press, Chicago, Illimois.

Levin S. A. and C. Goodyear (1980). Analysis of an age-structured fishery model. J. Math. Biol. 9:245-274.

Levin, S. A., and Castillo-Chavez, C. (1990). Topics in Evolutionary Biology. In: Mathematical and Statistical Developments of Evolutionary Theory (S. Lessard, ed.), pp. 327-358. NATO ASI Series, Kluwer Academic Publishers, Dordrecht, Boston, London.

Levin S. A. and Pimentel D. (1981). Selection of intermediate rates of increase in parasite-host systems. Am. Nat. 117:308-315.

Lotka, A.J. (1922). The stability of the normal age distribution. Proc. National Academy of Sciences 8 , 339-345. 
Lotka, A. J. (1923). Contributions to the anlysis of malaria epidemiology. Amer. J. Hygiene, 3, Jan. Supplement.

Lubkin, S. and C. Castillo-Chavez. (1993). A pair-formation approach to modeling inheritance of social traits. In: Proc. First World Congress on Nonlinear Analysts, Tampa, Florida, August 19-26, 1992. In press.

Luo, X. and C. Castillo-Chavez. (1991). Limit behavior of pair formation for a large dissolution rate. J. Math. Sys. Estim. Control 3, 247-264.

MacKendrick, A. G. (1926). Applications of mathematics to medical problems. In: Proc. Edinburgh Math. Soc. 44, 98-130.

May R. M. (1974). Biological Populations with non-overlapping generations: stable points, stable cycles, and chaos. J. Theor. Biol. 51:511-524.

May R. M. (1976). Simple mathematical models with very complicated dynamics. Nature 261:459467.

May R. M. (1985). Regulation of populations with nonoverlapping generations by microparasites: A purely chaotic system. Am. Nat. 125:573-584.

May R. M. (1987). Chaos and the dynamics of biological populations. Proc. Roy. Soc. London Ser. A 413:27-44.

May R. M. and Anderson R. M. (1983a). Epidemiology and genetics in the coevolution of parasites and hosts. Proc. Roy. Soc. London Ser. B 219:281-313.

May R. M. and Anderson R. M. (1983b). Parasite-host coevolution. In: Coevolution, (ed. D. Futuyma and M. Slatkin), 186-206. Sinauer, Sunderland, Massachusetts.

May, R.M. and R.M. Anderson: (1989). The transmission dynamics of human immunodeficiency virus (HIV). Phil. Trans. R. Soc. London B 321, 565-607.

May R. M. and Oster G. F. (1976). Bifurcations and dynamic complexity in simple ecological models. Am. Nat. 110:573-599.

McFarland, D.D. (1972). Comparison of alternative marriage models. In: Population Dynamics (T.N.E. Greville, ed.), pp. 89-106. Academic Press: New York, London.

Neubert M. G. and Kot M. (1992). The subcritical collapse of predator-prey models. Math. Biosci. 110:45-66.

Nold, A. (1980). Heterogeneity in disease-transmission modeling. Math. Biosci. 52, 227-240.

Olsen L. F. and Schaffer W. M. (1990). Chaos versus noisy periodicity: alternative hypotheses for childhood epidemics. Science 249:499-504.

Parlett, B. (1972). Can there be a marriage function? In: Population Dynamics (T.N.E. Greville, ed.), pp. 107-135. Academic Press: New York, London.

Pickering, J., J. A. Wiley, N. S. Padian, L. E. Lieb, D. F. Echenberg and J. Walker. (1986). Modelling the incidence of acquired immunodeficiency syndrome (AIDS) in San Francisco, Los Angeles and 
New York. Mathematical Modelling, 7:661-698.

Pollard, J.H. (1973). Models for the Growth of Human Populations. Cambridge University Press: Cambridge.

Pugliese, A. (1990a). Population models for disease with no recovery. J. Math. Biol. 28:65-82.

Pugliese, A. (1990b). An $\mathrm{S} \rightarrow \mathrm{E} \rightarrow \mathrm{I}$ epidemic model with varying population size. In: Proceedings of the International Conference on Differential Equations and Applications to Biology and Population Dynamics (S. Busenberg and M. Martelli, eds.), Lecture Notes in Biomathematics, 93:121-138, Springer-Verlag.

Ross, R. (1911). The Prevention of Malaria (2nd ed., with addendum). John Murray: London.

Rubin, G., Umbauch, D., Shyu, S-F., and Castillo-Chavez, C. (1992) Application of capture-recapture methodology to estimation of size of population at risk of AIDS and/or other sexuallytransmitted diseases. Statistics in Medicine. 11: 1533-1549.

Sattenspiel, L. (1987a). Population structure and the spread of disease. Human Biology 59, 411-438.

Sattenspiel, L. (1987b). Epidemics in nonrandomly mixing populations: a simulation. American Journal of Physical Anthropology, 73, 251-265.

Sattenspiel, L. and C.P. Simon. (1988). The spread and persistence of infectious diseases in structured populations. Math. Biosci. 90, 341-366.

Sattenspiel, L. and Castillo-Chavez, C. (1990). Environmental context, social interactions, and the spread of HIV. American J. of Human Biology 2: 397-417.

Schaffer W. M. (1985a). Can nonlinear dynamics elucidate mechanisms in ecology and epidemiology? IMA J. Math. Appl. Med. Biol. 2:221-252.

Schaffer W. M. (1985b). Order and chaos in ecological systems. Ecology 66:93-106.

Schaffer W. M. and Kot M. (1985). Nearly one dimensional dynamics in an epidemic. J. Theor. Biol. 112:403-427.

Schaffer W. M., Olsen L. F., Truty G. L.,a nd Fulmer S. L. (1990). The case of chaos in childhood epidemics. The Ubiquity of Chaos (Krasner S. ed.) Washington D.C. Am.Assoc. Advance. Sci. 138-166.

Soper, H. E. (1929). Interpretation of periodicity in disease prevalence, J. Roy. Statist. Soc. B 92:3473.

Takeuchi Y and Adachi N. (1983). Existence and bifurcation of stable equilibrium in two-prey, one predator communities. Bull. Math. Biol. 45:877-900.

Thieme, H. R. and Castillo-Chavez, C. (1989). On the role of variable infectivity in the dynamics of the human immunodeficiency virus epidemic. In: Mathematical and statistical approaches to AIDS epidemiology (C. Castillo-Chavez, ed.), pp. 157-176. Lecture Notes in Biomathematics 83, Springer-Verlag, Berlin, Heidelberg, New York, London, Paris, Tokyo, Hong Kong. 
Thieme, H. R. and Castillo-Chavez, C. How may infection-age dependent infectivity affect the dynamics of HIV/AIDS? SIAM J. Applied Math. (in press).

Velasco-Hernandez, J.X. and C. Castillo-Chavez. (1993). Modeling vector-host disease transmission and food web dynamics through the mixing/pair-formation approach. In: Proc. First World Congress on Nonlinear Analysts, Tampa, Florida, August 19-26, 1992. (In press).

Waldstätter, R. (1989). Pair formation in sexually transmitted diseases. In (C. Castillo-Chavez, ed.) Mathematical and Statistical Approaches to AIDS Epidemiology. Lecture Notes in Biomathematics 83, Springer-Verlag, Berlin, Heidelberg, New York, London, Paris, Tokyo, Hong Kong: 260-274. 
Figure 1. Kermack-MacKendrick Model. Phase Plot with Basic Reproductive Number = 3 (Runge-Kutta 2, fixed time-step solution).

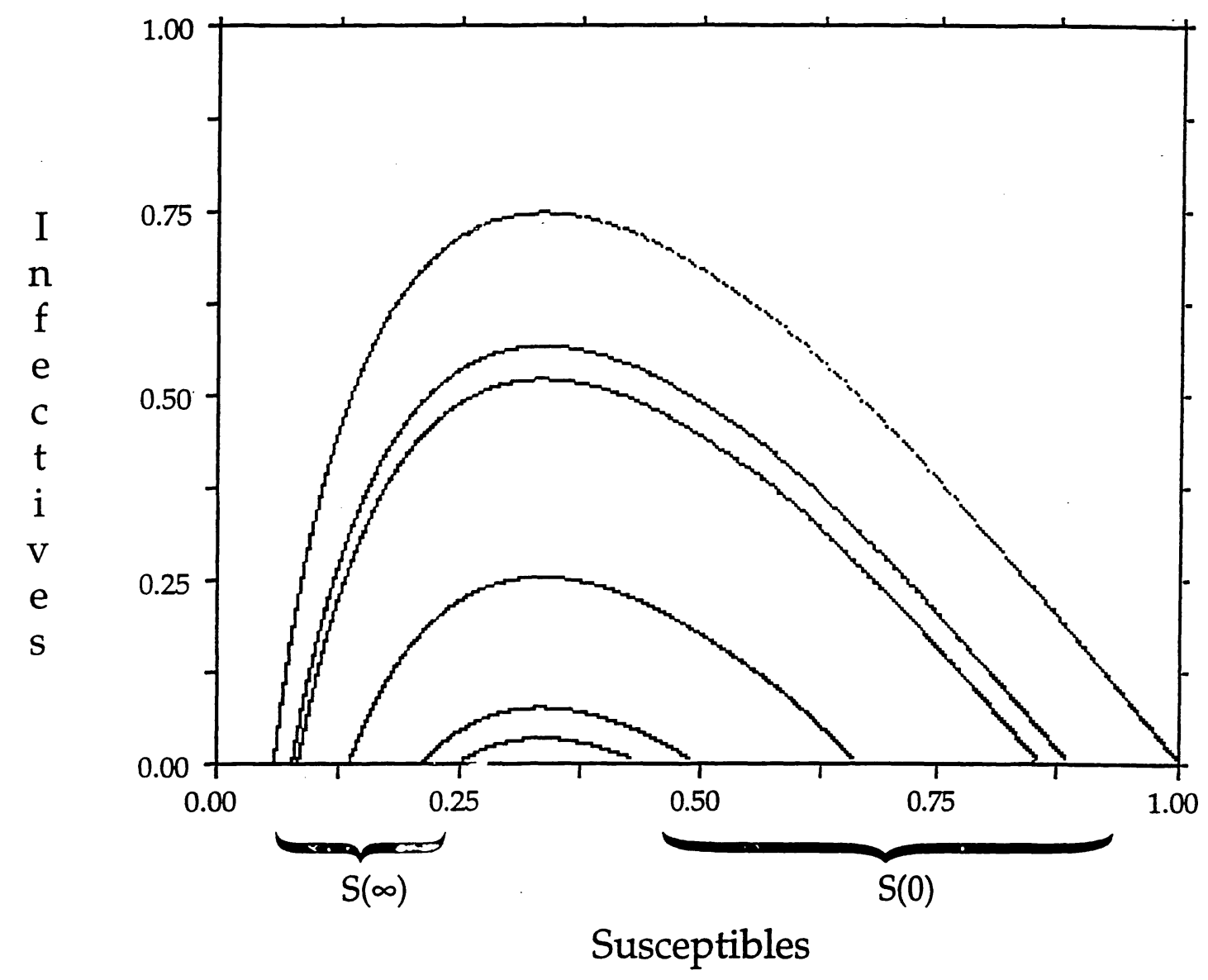

$S(0)$ denotes the initial proportion of susceptibles, while $S(\infty)$ denotes the susceptible proportion after the epizootic event. 
Figure 2. Real Mixing Matrices for Males and Females

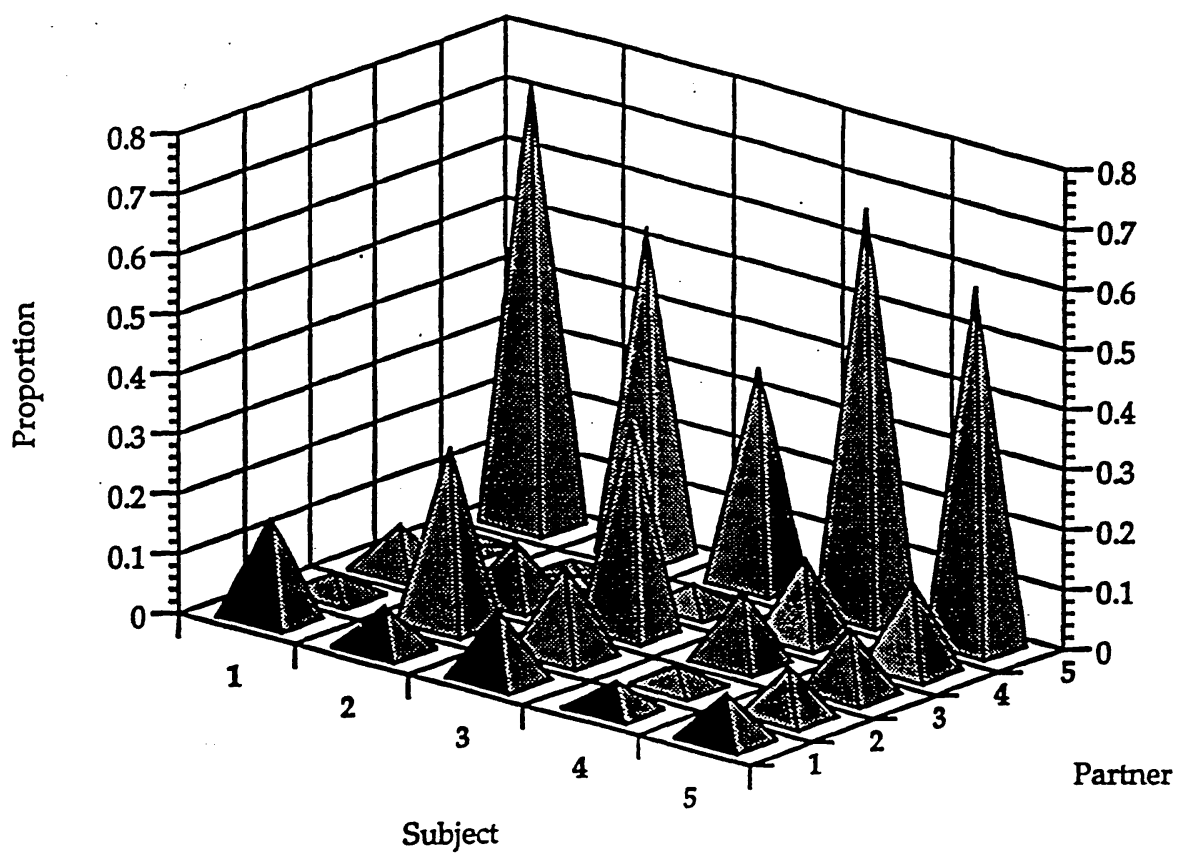

Data (male respondents): completed mixing matrix

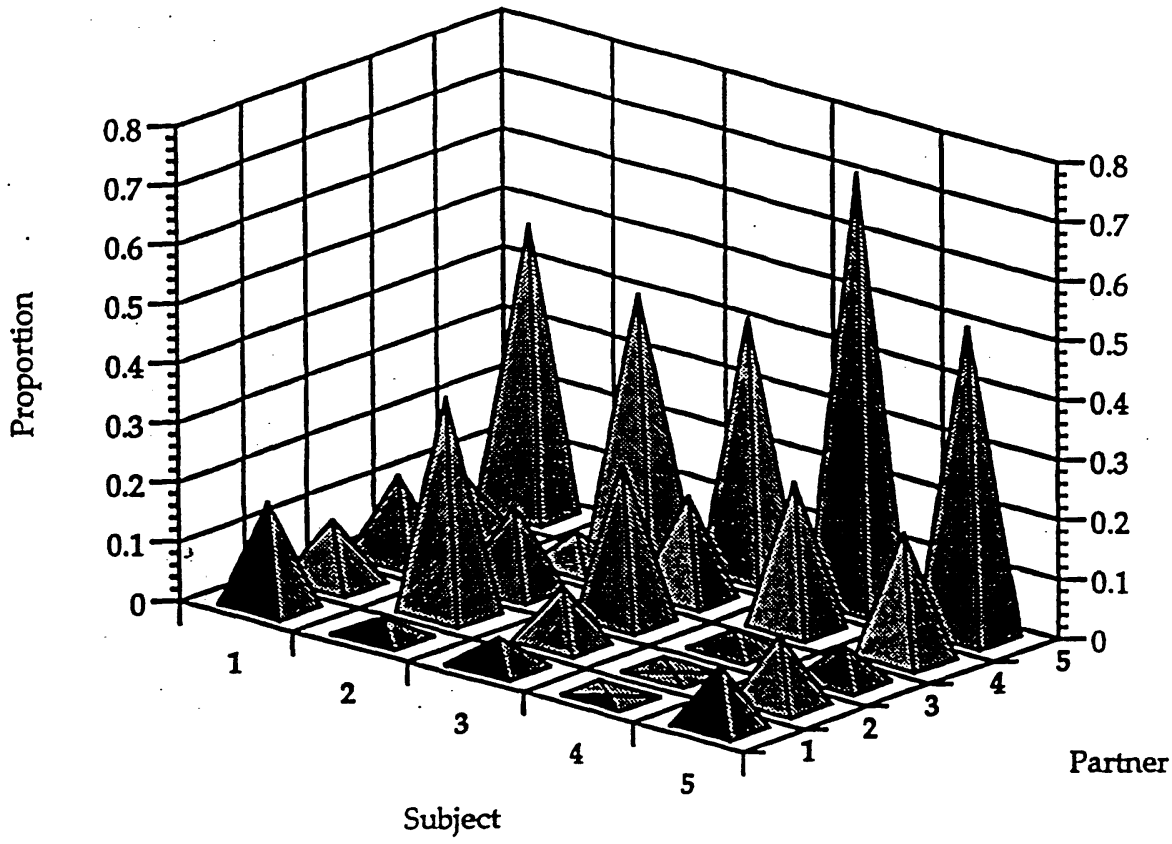

Data (female respondents): completed mixing matrix 
Figure 3. Ross Solution for Males and Females

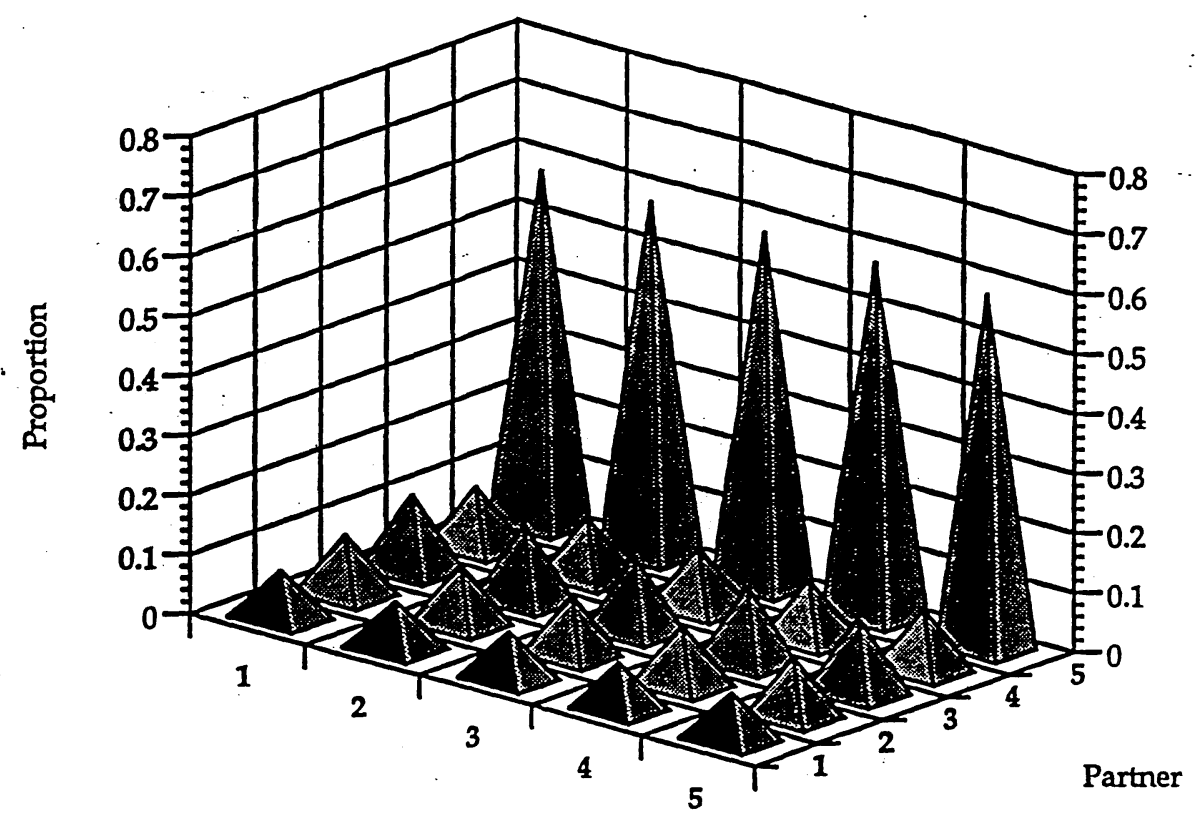

Subject

Ross solution for males

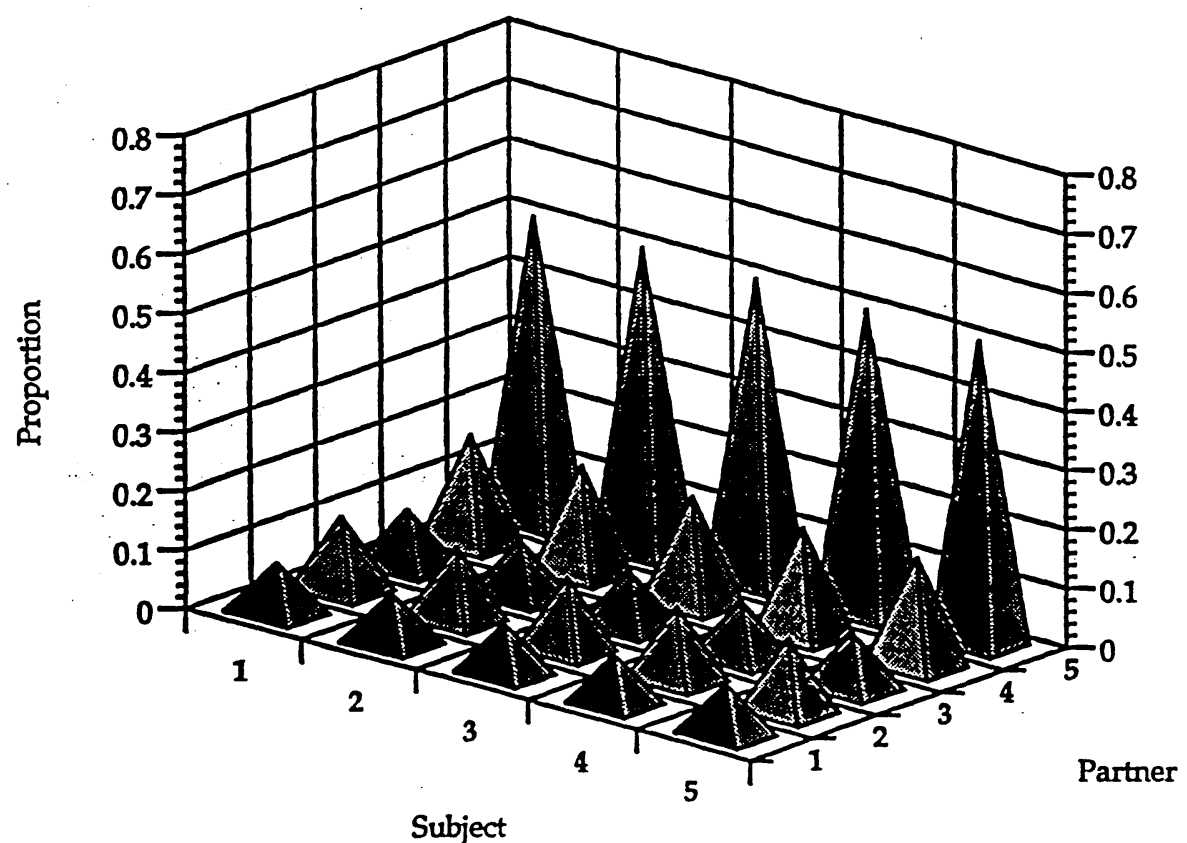

Subject

Ross solution for females 